\title{
Unusual Features of the Membrane Kinome of Trypanosoma brucei
}

$5 \quad{ }^{a}$ Center for Global Infectious Disease Research, Seattle Children's Research Institute, 307 Westlake Ave.

$6 \quad$ N., Seattle, WA 98109

7 bSeattle Biomedical Research Institute, 307 Westlake Ave. N, Seattle, WA 98109\#

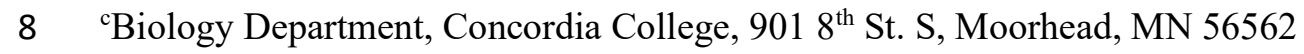

$9{ }^{\mathrm{d}}$ Department of Molecular Microbiology and Immunology, Johns Hopkins School of Public Health, 10 615N. Wolfe Street, Baltimore, MD 21205

11 eDepartments of Pediatrics and Global Health, University of Washington, Seattle, WA, 98195

$12 \%$ current address: Adaptive Biotechnologies, 1551 Eastlake Ave E, Suite 200. Seattle WA, 98102

$13 *$ corresponding author

14 \#Seattle Biomedical Research Institute merged with Seattle Children's Research Institute in 2018 


\section{Abstract}

In many eukaryotes, multiple protein kinases are situated in the plasma membrane where they respond to extracellular ligands. Ligand binding elicits a signal that is transmitted across the membrane, leading to activation of the cytosolic kinase domain. Humans have over 100 receptor protein kinases. In contrast, our search of the Trypanosoma brucei kinome showed that there were only ten protein kinases with predicted transmembrane domains, and unlike other eukaryotic transmembrane kinases, seven are predicted to bear multiple transmembrane domains. Most of the ten kinases, including their transmembrane domains, are conserved in both Trypanosoma cruzi and Leishmania species. Several possess accessory domains, such as Kelch, nucleotide cyclase, and forkhead-associated domains. Surprisingly, two contain multiple regions with predicted structural similarity to domains in bacterial signaling proteins. A few of the protein kinases have previously been localized to subcellular structures such as endosomes or lipid bodies. We examine here the localization of epitope-tagged versions of seven of the predicted transmembrane kinases in T. brucei bloodstream forms and show that five localized to the endoplasmic reticulum. The last two kinases are integral membrane proteins associated with the flagellum, flagellar pocket, or adjacent structures, as shown by both fluorescence and immunoelectron microscopy. Thus, these kinases are positioned in structures suggesting participation in signal transduction from the external environment.

Introduction

With its complex life cycle spanning different hosts and tissues, the response of Trypanosoma brucei to its environment is a subject of considerable interest. In other eukaryotes, signaling by extracellular molecules is initiated by ligand binding, typically to proteins situated at the plasma membrane. The most prominent categories of such proteins are G-protein coupled receptors (absent in trypanosomatids) and protein kinases (PKs) [1]. T. brucei also possesses a large family of surface proteins that have external ligand-binding domains and internal adenylate cyclase domains [2], although the ligands and signaling pathways involved remain to be elucidated. Transporters can also function in signal transduction by internalizing regulatory molecules such as Pi or cGMP [3-5]. In T. brucei, a plasma membrane carboxylate transporter is important in regulating the transition of mammalian bloodstream forms (BF) to the insect procyclic form (PF) stage [6]. catalytic domain, T. brucei has $\sim 180$ protein-coding regions that bear a canonical ePK domain [7]. Almost all PK orthologues are shared among T. brucei, Trypanosoma cruzi and Leishmania major. Most eukaryotes have tens to hundreds of PKs that bear transmembrane domains (TMDs), and in metazoans 
domain generates a signal that is transduced to the cytosolic catalytic domain resulting in kinase activation. In a previous analysis of the genome, we identified ten PKs predicted to bear TMDs as predicted by TMHMM [7], The redrawing of CDS boundaries based on 5' transcript mapping eliminated the putative TMD of one (Tb927.10.16160) and annotation of another PK was updated to include a TMD (Tb927.10.1910). The catalytic domains of these PKs cluster with serine/threonine kinases [7].

About half of the T. brucei TMD PKs have been characterized functionally. Tb927.2.2720, dubbed MEKK1 (so-named for the similarity of its catalytic domain to MAP kinase kinase kinases), is required for the quorum sensing pathway that triggers proliferating slender BF to enter stationary phase as a prelude to transmission [8]. Tb927.11.14070, known as $R D K 1$, encodes a repressor of $\mathrm{BF}$ to $\mathrm{PF}$ differentiation [9]. Tb927.11.8940 encodes LDK (lipid droplet kinase), which is present on lipid droplets and essential for their biogenesis [10]. Tb927.4.2500, which encodes an eIF2 kinase-related protein, EIF2K2K, is localized to the flagellar pocket [11]. While the function of this kinase in T. brucei is not known, its orthologue in Leishmania appears to regulate promastigote to amastigote development [12] and the $T$. cruzi orthologue similarly regulates the development of infective epimastigote forms [13]. Tb927.10.1910 is suggested to play a role in melarsoprol sensitivity on the basis of high-throughput RNAi assays [14]. Cell death was not observed upon depletion of the TM kinases in BF in a kinome-wide stem-loop RNAi study, although the degree of knockdown was not quantitated [9]. the presence of multiple TMDs, nucleotide cyclase domains, or regions of structural similarity to bacterial signaling modules. We examined the subcellular localization of seven of these PKs in monomorphic BF, which are unable to differentiate to PF. Five of the tagged PKs localized predominantly to the endoplasmic reticulum (ER), indicating they entered the secretory system. Two were found to have particularly distinctive distributions: FHK localized to a region at or near the flagellar pocket and MEKK1 localized similarly, as well as the flagellum. Immunoelectron microscopy of cultured PF confirmed the unique positioning of these two kinases.

\section{Results}

75 Domain structure. Figure 1A depicts schematics of the ten T. brucei PKs that have TMDs, showing the 76 location of the TMDs. Also shown are the PK catalytic domains and additional domains listed on

77 TriTrypDB (based primarily on sequence similarities), plus regions HHpred identified as having predicted 78 structural similarities to other protein modules. These PKs are large, with the smallest (the lipid droplet 79 kinase LDK) being 553 aa, about twice the size of the PK domain, and the rest ranging from 929 to 1678

80 aa. The PK domain of all of these kinases, with the exception of LDK, resides at the C-terminus. 
81 Transmembrane domains and signal peptides. Surprisingly, seven of the ten proteins are predicted by

82 TMHMM to be multi-pass proteins, having two or more TMDs, a topology that is extremely uncommon

83 for eukaryotic PKs. We therefore re-examined the TMD (predicted by TMHMM and shown TriTrypDB,

84 red bars in Fig. 1) using CCTOP (Constrained Consensus TOPology Prediction,

85 http://cctop.enzim.ttk.mta.hu/, which combines ten algorithms with structural data to yield a model. The

86 CCTOP models concurred with TMHMM for four PKs and proposed additional TMDs for the rest of the

87 PKs (Fig. 1, red arrowheads), and rejected one of the TMDs on RDK1 (red x). Some of the additional

88 TMDs predicted with CCTOP are unlikely to be authentic since they occur in the middle of domains, for

89 example, one that punctuates the ePK catalytic domain in Tb927.5.3150, and those within structural

90 domains of RDK1 and Tb927.9.3120. When we examined the orthologues of these T. brucei PKs in $L$.

91 major and T. cruzi, the number and position of the TMHMM-predicted TMDs generally concurred (Table

92 S1). The exceptions included the L. major orthologue of eIF2K2 (LmjF.34.2150), the T. cruzi CL-Brener

93 orthologues of Tb927.5.3150, and the T. cruzi orthologue of LDK (TcCLB.511801.14), all of which lack

94 a TMHMM-predicted TMD. However, CCTOP did predict TMDs in these proteins. Furthermore, since

95 LDK resides in a monolayer membrane surrounding lipid droplets rather than a bilayer, algorithms may

96 be less proficient at identifying relevant membrane insertion/association sequences. While it is not

97 possible to be certain of the number of TMDs from bioinformatic predictions, an even number of TMDs

98 would place the N- terminal region and C-terminal catalytic domain in the same compartment. For

99 example, MEKK1 is predicted to have a hairpin of two TMDs, separated by only four aa. The extended

$100 \mathrm{~N}$ - and C-regions would either both be cytosolic, or both be extracellular. We are unaware of any

101 transmembrane kinases that display catalytic domains on the external face of the plasma membrane, so

102 propose that the former possibility is most likely.

103 Most membrane proteins destined for the secretory system or plasma membrane enter the ER membrane

104 co-translationally, courtesy of a signal peptide or a transmembrane domain [15]. Interestingly, it has been

105 proposed that two TMDs separated by loop, such as seen in MEKK1 and several other TMD kinases (Fig.

106 1), may be a topogenic signal for insertion into the ER membrane [16]. Two of the PKs bearing TMDs are

107 also predicted to bear signal peptides: eIF2K2 and Tb927.5.3150. Three additional T. brucei PKs are

108 annotated as bearing signal peptides, but they lack predicted TMDs.

109 Domains identified by sequence similarity. Searches based on sequence similarity showed that

110 Tb927.5.3150 bears two Kelch/Kelch-like domains, which often participate in protein-protein

111 interactions. Additional domains related to signaling functions were detected on several PKs. Three bear

112 nucleotide cyclase-like domains (Tb927.3.5650, Tb927.9.12400, and Tb927.10.1910). Interestingly these

113 are the only T. brucei PKs identified as having such domains, although the parasite genome encodes 
multiple adenylyl cyclases with cytoplasmic catalytic domains and presumed ligand-binding extracellular domains [17]. One PK (Tb927.9.3120) contains a region related to PAS (Per-Arnt-Sim) domains, which usually function to bind small molecules or proteins intracellularly. Forkhead kinase, FHK,

117 Tb927.7.5220) is so-named for the presence of a forkhead-associated domain; these domains typically

118 interact with phosphopeptides (usually phosphothreonine in specific contexts) [18].

119 Conserved folds identified by structural predictions. The PKs were also subjected to HHpred analysis to

120 identify regions with predicted structural similarity to protein structures available in the protein database

121 PDB [19] (Fig. 1). The domains mentioned above all showed numerous matches with the related domains

122 on other proteins, with probability scores of over 99\%. A PAS-like region on FHK was additionally

123 identified by HHpred, with multiple hits having a probability above $90 \%$.

124 Surprisingly, two PKs have large regions of predicted structural similarity to molecular signaling modules

125 present in bacteria. RDK1 includes two regions with predicted structural similarity to other signaling

126 molecules (Fig. S1). The first is similar to bacterial sensor/receptor domains, such the ligand binding

127 domain of Pseudomonas aeruginosa histamine receptor TlpQ [20] and the extracellular domains of a set

128 of bacterial histidine kinases for which the ligands are unknown [21]. This region is followed by a

129 predicted TMD and then a segment predicted to fold similarly to full-length catalytic domains of several

130 bacterial and eukaryotic adenylyl/guanylyl cyclases. The cyclase region is followed by the eukaryotic PK

131 catalytic domain. This organization suggests that the sensor-like domain lies on the opposite side of the

132 membrane to the cyclase and PK domains. The modular organization of RDK1 is conserved in other

133 trypanosomatids, as well as the more distantly related free-living bodonid Bodo saltans [22].

134 Tb927.9.3120 has co-linear regions predicted to fold similarly to molecules involved in bacterial

135 signaling: histidine kinases and response regulators. The histidine kinases typically contain sensing

136 modules, followed by DHp dimerization regions, then catalytic/ATP binding domains (which

137 phosphorylate a histidine on DHp). Response regulators include receiver domains (which receive the

138 phosphate from DHp) and effector domains. In some cases, the kinase and receiver reside on the same

139 molecule (hybrid histidine kinases), although two component systems are more common. Importantly, in

$140 \mathrm{~Tb} 927.9 .3120$ the regions corresponding to each domain are essentially complete and ordered as in hybrid

141 bacterial histidine kinases [23, 24] (see Fig. 2). This organization of a hybrid histidine kinase cassette

142 followed by a eukaryotic protein kinase domain is conserved in the Tb927.9.3120 orthologues in other

143 trypanosomatids, such as T. cruzi and L. major, and the bodonid Bodo saltans (data not shown). Together,

144 this provides intriguing evidence of a bacterial genetic incursion of a signaling cassette into a common

145 ancestor of bodonids and trypanosomatids. Accordingly, we propose to name this gene dual ancestor

146 kinase $(D A K)$. 
147 Expression. We examined the expression of these PKs using our previous genome-wide ribosome

148 profiling data from pleiomorphic slender form BF (isolated from mice) and cultured PF [25]. Ribosome

149 profiling provides a direct measure of protein production. In Fig. 3, the dark blue and red bars show the

150 abundance of ribosome-associated mRNA footprints of BF and PF respectively. For comparison, the

151 mRNA abundances are also shown (light blue and pink bars). Several of the PKs show higher protein

152 production in slender BF than in PF, with MEKK1 showing 3-fold more expression in slender BF and

153 eIF2Ka having 2-fold more. However, the most dramatic difference was for RDK1 with 7-fold more

154 mRNA and 40-fold more protein production in slender BF than in PF. Conversely, Tb927.9.3120 had

155 about twice as much protein production in PF than slender BF, although the mRNA levels are very

156 similar between stages. Most of the PKs were close to the median level of protein production for all non-

157 pseudogenes (224 reads per $\mathrm{kb}$ in BF and 202 in PF). However, three PKs showed lower protein

158 production, being in the lowest quartile of all PKs. LDK protein production was low in both stages; FHK

159 protein production was low in BF and very low in PF; and Tb927.9.12400 protein production was almost

160 absent in BF and very low in PF.

161 Subcellular localization. Previous work localized T. brucei LDK to the lipid droplet membrane [10] and

162 eIF2K2 to the flagellar pocket [11] (and its T. cruzi orthologue to endosomes [13]). Additionally, the

163 protein encoded by Tb927.10.1910 was localized to the cytoplasm and endocytic organelles in PF in the

164 TrypTag project [26]. We determined the localization of C-terminally tagged versions of the remaining

165 transmembrane PKs in single marker strain BF. A western blot (Fig. S2) shows the migration of the

166 tagged proteins in SDS gels, which corresponded reasonably well to the predicted sizes given in Table S1.

167 In the absence of antibodies specific to individual PKs, it is not possible to predict whether the expression

168 levels of the tagged protein are comparable to the endogenous levels. However, the very low levels of

169 FHK and Tb927.9.12400 revealed by ribosome profiling compared to their ready detection by western

170 blot (Fig. 3) suggest that the tagged versions of those PKs may be over-expressed.

171 Many tagged TMD PKs localize to the ER. As summarized in Table 1 and shown in Fig. 4, five of the

172 tagged PKs with predicted TMDs colocalized with the ER marker BiP. None of these PKs is classified as

173 related to the mammalian ER kinase PEK [7]. Staining of the tagged proteins is evident around the body

174 of the cell but does not extend along the flagellum after it passes the anterior end of the cell body. As with

$175 \mathrm{BiP}$, some perinuclear staining is also seen; in most cases this is fainter than the peripheral staining.

176 RDK1 was previously shown to be associated with membranes and localized to the periphery of the

177 parasite [9]; our colocalization studies indicate that this peripheral localization is the ER. While it is clear

178 that these PKs enter the secretory system, the ER may not be their final destination, which could be

179 masked by potential over-expression. 
MEKK1 is an integral membrane protein of the flagellum and flagellar pocket with kinase activity. Immunofluorescence analysis showed that MEKK1-V5 is present along the flagellum, where it aligns with PIFTC3, a component of the flagellar protein transport system [27] and is clearly distinct from the flagellar $\mathrm{K}+$ channel that also extends along the flagellum [28]. We also observed staining adjacent to the kinetoplast, which was distinct from, but adjacent to, the depot of PIFTC3. When two kinetoplasts were seen, two puncta of both PIFTC3 and MEKK1 were visible. Immunoelectron microscopy was performed using PF expressing MEKK1 C-terminally tagged with HA epitopes. As shown in Fig. 6, gold particle staining was present at the flagellar pocket (A, B) but not at the kinetoplast (C). In a dividing cell, both flagellar pockets were stained. MEKK1-HA was also seen along the flagellum (Fig 6E). The MEKK1-HA at the flagellar pocket may represent molecules in transit to the flagellum or a final destination, as is seen for PIFTC3 [27] and (Figure 5).

To assess membrane association, we conducted cell extraction and fractionation of PF expressing MEKK1-HA. The full-length protein migrated anomalously, somewhat faster than the $220 \mathrm{kDa}$ marker (the tagged protein is predicted to be $167 \mathrm{kDa}$ ). Membrane proteins are known to exhibit anomalous migration on SDS-PAGE gels, migrating either slower or faster than predicted, with apparent molecular masses averaging $80-113 \%$ of the predicted value [29]. The $\sim 95 \mathrm{kDa}$ V5-tagged fragment can be disregarded since it is not seen in cells lysed directly in SDS sample buffer, and therefore represents a degradation fragment. As shown in the immunoblot in Fig. 7, upon treatment of PF with digitonin to release the cytosol, the intact kinase remained in the pellet fraction (P1). Subsequent treatment of the digitonin pellet with sodium carbonate, $\mathrm{pH}$ 11, showed MEKK-V5 in the pellet (P2), demonstrating that it is an integral membrane protein. Marker proteins PGKB (cytosolic), PGKA (glycosomal which shows

201 partial membrane association) [30] and vacuolar pyrophosphatase, TcVSP) (integral vacuolar membrane)

$202[31]$ verified the fractionation procedure.

203 To test for catalytic activity, anti-V5 immunoprecipitates from PF expressing MEKK1-V5, as well as 204 from the untransfected parental 29.13 line, were incubated with the exogenous substrate myelin basic protein (MBP) in the presence of $\gamma^{32} \mathrm{P}-\mathrm{ATP}$. The proteins were then separated by SDS-PAGE and analyzed by phosphorimaging (Fig. 7B, top) and western blot (Fig. 7B, bottom). MBP was

207 phosphorylated in the sample containing MEKK1-V5, but not the untransfected control, thereby

208 demonstrating that MEKK1 can phosphorylate an exogenous substrate.

209 FHK is an integral membrane protein of the flagellar pocket with kinase activity. Like MEKK1, FHK-V5 210 demonstrated staining near the kinetoplast, close to PIFTC3 upon immunofluorescence analysis of BF 211 (Fig. 8A). However, staining did not extend along the flagellum and little co-localization with BiP was 212 observed (Fig. 8B). Live PF expressing MEKK1-HA were incubated with biotinylated tomato lectin at $4^{\circ}$ 
$213 \mathrm{C}$ and then fixed for anti-HA staining (Fig. 8C). The low temperature allows lectin binding to the flagellar

214 pocket without internalization into the parasite. The HA staining was adjacent to and possibly overlapping

215 the tomato lectin signal. Fig. 8D depicts a PF parasite stained for DNA and FHK. Individual deconvolved

216 planes showed FHK between the nucleus and kinetoplast in what appears to be a basket-like structure.

217 Immunoelectron microscopy of PF demonstrated that the protein was localized to the flagellar pocket but

218 not to the flagellum or kinetoplast (Fig. 9A, enlarged in 9a), and occasionally staining is seen more

219 prominently on one side of the flagellar pocket (Fig. 9B, enlarged in 9b). Sometimes staining was

220 observed on vesicles close to the flagellar pocket, but only sporadically outside the region of the pocket

221 (Fig. 9 C, D).

222 PF expressing FHK-HA were fractionated as described above for MEKK1. Full-length FHK-HA was in

223 the digitonin pellet, and the carbonate-insoluble fraction, indicating it is an integral membrane protein

224 (Fig. 10A). As with MEKK1, the smaller degradation fragment can be disregarded since it was absent

225 from cells lysed in SDS sample buffer. To test for catalytic activity, immunoprecipitates from PF

226 expressing FHK-HA, as well as the untransfected, parental single marker line, were assayed as described

227 for MEKK1. As shown by phosphorimaging (Fig. 10B, top) and western blot (Fig. 10B, bottom), MBP

228 was phosphorylated in the sample containing FHK-HA, but not the untransfected control, providing

229 evidence of FHK catalytic activity.

230 Discussion

231 A plethora of transmembrane domains

232 In contrast to metazoan kinomes, which have large numbers of TMD PKs, protozoan kinomes vary

233 widely in their representation of TMD PKs. At one extreme, the Giardia lamblia genome encodes only

234 two predicted transmembrane PKs (two other PKs have predicted TMDs unlikely to be authentic since

235 they punctuate the catalytic domain). At the other extreme is Entamoeba histolytica with more than 80

236 predicted TMD PKs, most of which are related at the sequence level and some of which are likely non-

237 catalytic [32]. Like E. histolytica, Plasmodium falciparum has evolved a unique set of PKs, the FIKK

238 kinases, that comprise the majority of the parasite's 17 PKs with predicted TMDs. The FIKK kinases bear

239 the signature sequence for export out of the parasite [33], and at least some of them are important in

240 remodeling the host erythrocyte [34,35]. Like P. falciparum, trypanosomatids fall between extremes of

241 G. lamblia and E. histolytica, with a paucity of PKs that bear TMDs compared to metazoans. These

242 trypanosomatid PKs are classified firmly with serine/threonine kinases as opposed to tyrosine kinases

243 characteristic of transmembrane PKs of animals [7]. Just as interesting, most of these trypanosomatid PKs

244 are predicted to have multiple membrane spanning domains, a feature that is extremely rare in eukaryotes. 
To our knowledge, a single ePK domain protein in eukaryotes has been biochemically demonstrated to have more than one TMD, lemur tail kinase 2 [36], but other members of that family of PKs are also predicted to have two TMDs [37]. Interestingly, in perusing the E. histolytica genome database, we noted a few PKs with two likely TMDs. Another interesting observation is that many bacterial histidine kinases with several TMDs have been identified in genome-wide bioinformatic analyses [38]. These include the 5-TMD bearing Staphylococcus aureus LytS histidine kinase which acts as a sensor of membrane potential [39]. Another example is Bacillus subtilis DesK, in which TMDs are involved in signaling temperature-induced changes in membrane fluidity [40]. Thus, TMDs can modulate kinase activity in response to environmental changes even when no extracellular ligand binding domains are present. It will be interesting to determine if any of the TMDS in the T. brucei PKs examined here have such effects.

\section{Flagellar and flagellar pocket localization and function}

The localization of MEKK1 and FHK to the flagellum and flagellar pocket respectively raises the question of what biochemical processes they may regulate. Initiation of stumpy development appears to be triggered by the uptake of oligopeptides by the transporter GPR89 [41]. MEKK1 acts early in the developmental pathway, which involves multiple additional kinases [8]. How the oligopeptide signal intersects with MEKK1 is not clear given that GPR89 is distributed across the plasma membrane and is not specifically localized to, or perhaps not present at all, at the flagellar membrane. However, other molecules that may be involved in development are also localized to the flagella, such as adenylate cyclases and phosphodiesterases that are present on the flagellum, as well as a calcium channel [42]. endocytosis. Therefore, the localization of FHK to the flagellar pocket could suggest a function in these processes. RNAi data suggests that FHK is dispensable in BF [9, 43] (also corroborated by our preliminary data), so it is unlikely that it functions as a global regulator of such essential processes. Instead, its function may be focused on specific conditions (e.g., stress response) or certain pathways within the broader categories of exocytosis, endocytosis and recycling of membrane molecules. Because only the flagellar pocket is free of the densely packed, GPI-anchored variant surface protein coat which covers the surface of the parasite and bears many distinct proteins, FHK could also be positioned at the

272 flagellar pocket to sense interaction with transmembrane proteins or even to biophysical changes in this

273 distinct membrane domain, such as those sensed by LytS and DesK.

\section{Signaling domains of likely bacterial origin}

275 There are numerous genes in trypanosomatids, especially those encoding metabolic enzymes, which are 
277 providing evidence of lateral gene transfer. Our HHpred analysis showed that two T. brucei PKs have

278 regions predicted at $>95 \%$ probability to fold similarly to bacterial signaling modules. FHK has a region

279 resembling ligand-sensing domains found on chemotaxis proteins and certain histidine kinases, but not on

280 eukaryotic proteins. DAK has cassette bearing four regions common to hybrid histidine kinases: a sensor,

281 a dimerization/phosphoacceptor, a catalytic domain and a receiver domain. These modular structures are

282 conserved in genomes across trypanosomatids and in the bodonid Bodo saltans, ruling out an artifactual

283 explanation and arguing for a functional and possibly regulatory role. While this combination of domains

284 is unusual, Uniprot searches do identify predicted proteins that possess both an ePK domain and a

285 histidine kinase cassette. Most of these predicted proteins are bacterial, but some are found in eukaryotes

286 (predominantly fungi). Those in eukaryotes almost exclusively have hybrid type histidine kinase domains

287 (i.e, with a receiver domain on the same molecule) as is seen on DAK. Few (if any) of these histidine-ePK

288 kinases have been explored functionally to understand how the domains interact and influence the

289 signaling properties of the protein. DAK's potential for histidine kinase activity that phosphorylates the

290 DHp region appears low since the conserved histidine is mutated. However, the aspartic acid in the

291 receiver domain that accepts the phosphate is still present. Clearly additional work will need to be done to

292 understand the roles of the two ancestral kinase regions on the kinase activity and ultimately function of

293 DAK [25].

\section{Materials and Methods}

295 Parasites. The work described uses PF 29-13 and single marker BF lines, which are derivatives of the $T$.

296 brucei 427 strain [44]. Both lines express T7 RNA polymerase and the tetracycline (Tet) repressor,

297 allowing for Tet-regulated expression of transfected genes. PF were grown in SDM-79 (JRH Biosciences)

298 supplemented with $15 \%$ fetal calf serum containing $15 \mu \mathrm{g} / \mathrm{ml} \mathrm{G} 418$ and $50 \mu \mathrm{g} / \mathrm{ml}$ hygromycin to maintain

299 the T7 RNA polymerase and Tet repressor genes. BF were grown in HMI-9 with $2.5 \mu \mathrm{g} / \mathrm{ml} \mathrm{G418.}$

300 Plasmids were transfected into PF as described [44] and transfectants were selected with $1 \mu \mathrm{g} / \mathrm{ml}$

301 puromycin. BF were transfected as described [45] and modified by [46]. BF transfectants were selected

302 with either $5 \mu \mathrm{g} / \mathrm{ml}$ hygromycin or blastocidin $\mathrm{S}$ depending on the plasmid vector.

303 Plasmids and Cloning. For expression of V5-tagged proteins in BF, we generated the plasmid pT7-3V5-

304 Hyg by replacing the GFP and lacZ stuffer region of pT7-GFP (kindly supplied by David Horn) [47] with

305 the multicloning site and 3V5 epitopes derived from pLEW79-3V5-Pac [10]. Expression was driven by a

306 tetracycline (Tet) regulated T7 promoter and the 3' UTR was derived from aldolase. For expression of

307 HA-tagged proteins in PF, either pLEW79-3V5-PAC or plasmid pLEW100v5-3HA-BSD were utilized.

308 The latter was created by replacing the luciferase gene in pLEW100v5-BSD (kindly supplied by George 
Cross) with the multicloning sites derived from pLEW79-3V5-PAC and three HA epitope tags. Expression was driven by a tetracycline (Tet) regulated PARP promoter and the 3' UTR was derived from aldolase. The coding sequences for the PKs were amplified and PCR products were digested with the restriction enzymes noted in the primer and ligated into appropriately digested vectors. The expression construct for Tb927.9.3120 also included the endogenous splice acceptor. All primers are described in Table S2.

Western blots. Cell lysates were generated, and proteins resolved by SDS-PAGE and transferred to nitrocellulose as described [48]. V5-tagged proteins were detected with mouse monoclonal anti-V5 antibody (ThermoFisher) at $0.3 \mu \mathrm{g} / \mathrm{ml}$. HA-tagged proteins were detected with mouse (Covance) or rat (Roche) monoclonal anti-V5 antibodies at $1 \mu \mathrm{g} / \mathrm{ml}$ or $12.5 \mathrm{ng} / \mathrm{ml}$ respectively. Bound antibodies were revealed with IRDye $800 \mathrm{CW}$ dye-conjugated goat anti-mouse Ig (Li-COR) at $25 \mathrm{ng} / \mathrm{ml}$ and data was visualized on a Li-COR Odyssey.

Immunofluorescence analysis. IFAs were performed as previously described [48]. In brief, BF parasites were prefixed in $4 \%$ paraformaldehyde for $5 \mathrm{~min}$ on ice prior to placing on poly-L-lysine coverslips. The V5 epitope tag was detected by using mouse monoclonal anti-V5 antibody (Invitrogen) at $1 \mathrm{ug} / \mathrm{ml}$, followed by goat anti-mouse IgG conjugated with fluorescein isothiocyanate (FITC) (Southern Biotechnology). Additional antibodies used for colocalization included antibodies directed against BiP [49] (kindly supplied by Jay Bangs), used at 1:400; PIFTC3 [27] (kindly supplied by Elisabetta Ullu), used at 1:1000; TcCat [28] (kindly supplied by Roberto DeCampo), used at 1:250; YL1/2 (Gene-Tex), used at $1 \mu \mathrm{g} / \mathrm{ml}$. To visualize the flagellar pocket, pre-cooled cells were incubated at $4^{\circ} \mathrm{C}$ with biotinylated tomato lectin (Vector Laboratories) at $2 \mu \mathrm{g} / \mathrm{ml}$. After fixation, tomato lectin was visualized with $2 \mu \mathrm{g} / \mathrm{ml}$ streptavidin conjugated to either Texas Red or Alexa Fluor 488 (ThermoFisher). Dyeconjugated secondary antibodies (Southern Biotechnology) were used at $2 \mu \mathrm{g} / \mathrm{ml}$. Images were scaled to span the graph of signal intensity of the image field.

Immunoelectron microscopy. Preparations of parasites were fixed in 4\% paraformaldehyde (Electron Microscopy Sciences, PA) in 0.25 M HEPES ( $\mathrm{pH} 7.4$ ) for $1 \mathrm{~h}$ at room temperature, then in $8 \%$ paraformaldehyde in the same buffer overnight at $4 \mathrm{oC}$. They were infiltrated, frozen and sectioned as previously described [50]. The sections were immunolabeled with rat anti-HA antibodies (1:50 in PBS/1\% fish skin gelatin), then with anti-mouse IgG antibodies, followed directly by $10 \mathrm{~nm}$ protein Agold particles (Department of Cell Biology, Medical School, Utrecht University, the Netherlands) before examination with a Philips CM120 Electron Microscope (Eindhoven, the Netherlands) under $80 \mathrm{kV}$. 
340 Cell fractionation and Kinase assays. PF expressing HA-tagged proteins washed in PBS containing 10

$341 \mathrm{mM}$ glucose. They were permeabilized with digitonin and the organellar pellet was extracted with sodium

342 carbonate as described [51] except that Complete Protease tablets (Roche) were included for both

343 digitonin permeabilization and carbonate extraction, to prevent protein degradation.

344 Cell lysates from PF cells expressing either V5-tagged MEKK1 or HA-tagged FHK along with the

345 untransfected parental line 29.13 were prepared as described [52]. Epitope tagged proteins were

346 immunoprecipitated from cell lysate prepared from $10^{8}$ cells. Kinase assays were done as described [53]

347 using ${ }^{32} \mathrm{P} \gamma \mathrm{ATP}$ with myelin basic protein as an exogenous substrate. Reactions were resolved by SDS-

348 PAGE and transferred to nitrocellulose and labeled proteins detected by phosphorimaging. Blots were

349 subsequently probed with antibodies directed against either V5 or HA.

350 Acknowledgements: This work was supported in part by a grant 5R21AI101424 from the National

351 Institutes of Health and by Seattle Children's Research Institute.

352 Author contributions

353 Concept and experimental design were done by B.C.J and M.P. Experiments were performed by B.C.J,

354 P.V. and J.F except electron microscopy done by I.C. Manuscript was written by M.P. and B.C.J. All

355 authors have reviewed the manuscript.

356 Conflicts of interest

357 The authors declare no competing interest. 


\section{References}

1. $\quad$ El-Sayed, N.M., et al., The genome sequence of Trypanosoma cruzi, etiologic agent of Chagas disease. Science, 2005. 309(5733): p. 409-415.

2. Alexandre, S., et al., Families of adenylate cyclase genes in Trypanosoma brucei. Mol. Biochem. Parasitol, 1996. 77(2): p. 173-182.

3. Norouzi, S., et al., Zinc transporters and insulin resistance: therapeutic implications for type 2 diabetes and metabolic disease. J. Biomed. Sci, 2017. 24(1): p. 87.

4. Bon, N., et al., Phosphate (Pi)-regulated heterodimerization of the high-affinity sodiumdependent Pi transporters PiT1/Slc20a1 and PiT2/Slc20a2 underlies extracellular Pi sensing independently of Pi uptake. J. Biol Chem, 2017.

5. Henjakovic, M., et al., Human organic anion transporter 2 is distinct from organic anion transporters 1 and 3 with respect to transport function. Am. J. Physiol. Renal Physiol, 2015. 309(10): p. F843-F851.

6. Dean, S., et al., A surface transporter family conveys the trypanosome differentiation signal. Nature, 2009. 459(7244): p. 213-7.

7. Parsons, M., et al., Comparative analysis of the kinomes of three pathogenic trypanosomatids: Leishmania major, Trypanosoma brucei and Trypanosoma cruzi. BMC. Genomics, 2005. 6: p. 127.

8. McDonald, L., et al., Non-linear hierarchy of the quorum sensing signalling pathway in bloodstream form African trypanosomes. PLoS Pathog, 2018. 14(6): p. e1007145.

9. Jones, N.G., et al., Regulators of Trypanosoma brucei cell cycle progression and differentiation identified using a kinome-wide RNAi screen. PLoS Pathog, 2014. 10(1): p. e1003886.

10. Flaspohler, J.A., et al., A novel protein kinase localized to lipid droplets is required for droplet biogenesis in trypanosomes. Eukaryot. Cell, 2010. 9(11): p. 1702-1710.

11. Moraes, M.C., et al., A novel membrane-bound eIF2 alpha kinase in the flagellar pocket of Trypanosoma brucei. Eukaryot. Cell, 2007. 6: p. 1979-1991.

12. Chow, C., et al., Promastigote to amastigote differentiation of Leishmania is markedly delayed in the absence of PERK eIF2alpha kinase-dependent eIF2alpha phosphorylation. Cell Microbiol, 2011. 13(7): p. 1059-77.

13. da Silva Augusto, L., et al., A membrane-bound eIF2 alpha kinase located in endosomes is regulated by heme and controls differentiation and ROS levels in Trypanosoma cruzi. PLoS Pathog, 2015. 11(2): p. e1004618.

14. Alsford, S., et al., High-throughput decoding of antitrypanosomal drug efficacy and resistance. Nature, 2012. 482(7384): p. 232-6.

15. Shao, S. and R.S. Hegde, Membrane protein insertion at the endoplasmic reticulum. Annu Rev Cell Dev Biol, 2011. 27: p. 25-56.

16. Lee, H. and H. Kim, Membrane topology of transmembrane proteins: determinants and experimental tools. Biochem Biophys Res Commun, 2014. 453(2): p. 268-76.

17. Paindavoine, P., et al., A gene from the variant surface glycoprotein expression site encodes one of several transmembrane adenylate cyclases located on the flagellum of Trypanosoma brucei. Mol Cell Biol, 1992. 12(3): p. 1218-25.

18. Mahajan, A., et al., Structure and function of the phosphothreonine-specific FHA domain. Sci Signal, 2008. 1(51): p. re12.

19. Zimmermann, L., et al., A Completely Reimplemented MPI Bioinformatics Toolkit with a New HHpred Server at its Core. J Mol Biol, 2018. 430(15): p. 2237-2243.

20. Corral-Lugo, A., et al., High-Affinity Chemotaxis to Histamine Mediated by the TlpQ Chemoreceptor of the Human Pathogen Pseudomonas aeruginosa. mBio, 2018. 9(6).

21. Zhang, Z. and W.A. Hendrickson, Structural characterization of the predominant family of histidine kinase sensor domains. J Mol Biol, 2010. 400(3): p. 335-53. 
22. Jackson, A.P., et al., Kinetoplastid Phylogenomics Reveals the Evolutionary Innovations Associated with the Origins of Parasitism. Curr Biol, 2016. 26(2): p. 161-172.

23. Wang, C., et al., Mechanistic insights revealed by the crystal structure of a histidine kinase with signal transducer and sensor domains. PLoS Biol, 2013. 11(2): p. e1001493.

24. Dubey, B.N., et al., Hybrid histidine kinase activation by cyclic di-GMP-mediated domain liberation. Proc Natl Acad Sci U S A, 2020. 117(2): p. 1000-1008.

25. Jensen, B.C., et al., Extensive stage-regulation of translation revealed by ribosome profiling of Trypanosoma brucei. BMC Genomics, 2014. 15(1): p. 911.

26. Dean, S., J.D. Sunter, and R.J. Wheeler, TrypTag.org: A Trypanosome Genome-wide Protein Localisation Resource. Trends Parasitol, 2017. 33(2): p. 80-82.

27. Franklin, J.B. and E. Ullu, Biochemical analysis of PIFTC3, the Trypanosoma brucei orthologue of nematode DYF-13, reveals interactions with established and putative intraflagellar transport components. Mol. Microbiol, 2010. 78(1): p. 173-186.

28. Jimenez, V. and R. Docampo, Molecular and electrophysiological characterization of a novel cation channel of Trypanosoma cruzi. PLoS Pathog, 2012. 8(6): p. e1002750.

29. Rath, A. and C.M. Deber, Correction factors for membrane protein molecular weight readouts on sodium dodecyl sulfate-polyacrylamide gel electrophoresis. Anal Biochem, 2013. 434(1): p. 6772.

30. Parker, H.L., et al., Three genes and two isozymes: Gene conversion and the compartmentalization and expression of the phosphoglycerate kinases of Trypanosoma (Nannomonas) congolense. Mol. Biochem. Parasitol, 1995. 69: p. 269-279.

31. Galizzi, M., et al., Evidence for the role of vacuolar soluble pyrophosphatase and inorganic polyphosphate in Trypanosoma cruzi persistence. Mol Microbiol, 2013. 90(4): p. 699-715.

32. Beck, D.L., et al., Identification and gene expression analysis of a large family of transmembrane kinases related to the Gal/GalNAc lectin in Entamoeba histolytica. Eukaryot Cell, 2005. 4(4): p. 722-32.

33. Schneider, A.G. and O. Mercereau-Puijalon, A new Apicomplexa-specific protein kinase family: multiple members in Plasmodium falciparum, all with an export signature. BMC Genomics, 2005. 6(1): p. 30.

34. Davies, H., et al., An exported kinase family mediates species-specific erythrocyte remodelling and virulence in human malaria. Nat Microbiol, 2020. 5(6): p. 848-863.

35. Nunes, M.C., et al., Plasmodium falciparum FIKK kinase members target distinct components of the erythrocyte membrane. PLoS One, 2010. 5(7): p. e11747.

36. Nixon, A., et al., Determination of the membrane topology of lemur tyrosine kinase 2 (LMTK2) by fluorescence protease protection. Am J Physiol Cell Physiol, 2013. 304(2): p. C164-9.

37. Hisanaga, S.I., et al., LMTK1, a Novel Modulator of Endosomal Trafficking in Neurons. Front Mol Neurosci, 2020. 13: p. 112.

38. Anantharaman, V. and L. Aravind, Application of comparative genomics in the identification and analysis of novel families of membrane-associated receptors in bacteria. BMC Genomics, 2003. 4(1): p. 34.

39. Patel, K. and D. Golemi-Kotra, Signaling mechanism by the Staphylococcus aureus twocomponent system LytSR: role of acetyl phosphate in bypassing the cell membrane electrical potential sensor LytS. F1000Res, 2015. 4: p. 79.

40. Fernandez, P., et al., Transmembrane Prolines Mediate Signal Sensing and Decoding in Bacillus subtilis DesK Histidine Kinase. mBio, 2019. 10(6).

41. Rojas, F., et al., Oligopeptide Signaling through TbGPR89 Drives Trypanosome Quorum Sensing. Cell, 2019. 176(1-2): p. 306-317 e16.

42. Kelly, F.D., P.A. Yates, and S.M. Landfear, Nutrient sensing in Leishmania: Flagellum and cytosol. Mol Microbiol, 2020.

43. Fernandez-Cortes, F., et al., RNAi screening identifies Trypanosoma brucei stress response protein kinases required for survival in the mouse. Sci Rep, 2017. 7(1): p. 6156. 
44. Wirtz, E., et al., A tightly regulated inducible expression system for conditional gene knock-outs and dominant-negative genetics in Trypanosoma brucei. Mol Biochem Parasitol, 1999. 99(1): p. 89-101.

45. Carruthers, V.B. and G.A.M. Cross, High-efficiency clonal growth of bloodstream- and insectform Trypanosoma brucei on agarose plates. Proc. Natl. Acad. Sci. USA, 1992. 89: p. 8818-8821.

46. Schnaufer, A., et al., An RNA ligase essential for RNA editing and survival of the bloodstream form of Trypanosoma brucei. Science, 2001. 291(5511): p. 2159-2162.

47. Alsford, S., et al., Tagging a T. brucei RRNA locus improves stable transfection efficiency and circumvents inducible expression position effects. Mol Biochem Parasitol, 2005.

48. Jensen, B.C., et al., The NOG1 GTP-binding protein is required for biogenesis of the $60 \mathrm{~S}$ ribosomal subunit. J Biol Chem, 2003. 278: p. 32204-32211.

49. Bangs, J.D., et al., Molecular cloning and cellular localization of a BiP homologue in Trypanosoma brucei. Divergent ER retention signals in a lower eukaryote. J. Cell Sci, 1993. 105: p. 1101-1113.

50. Folsch, H., et al., Distribution and function of AP-1 clathrin adaptor complexes in polarized epithelial cells. J Cell Biol, 2001. 152(3): p. 595-606.

51. Saveria, T., et al., Conservation of PEX19-binding motifs required for protein targeting to mammalian peroxisomal and trypanosome glycosomal membranes. Eukaryot Cell, 2007. 6(8): p. 1439-1449.

52. Das, A., et al., Distinct domains of a nucleolar protein mediate protein kinase binding, interaction with nucleic acids and nucleolar localization. Journal of Cell Science, 1998. 111 ( Pt 17): p. 2615-2623.

53. Park, J.H., et al., Molecular cloning of Trypanosoma brucei CK2 catalytic subunits: the alpha isoform is nucleolar and phosphorylates the nucleolar protein Nopp44/46. Molecular and Biochemical Parasitology, 2002. 119(1): p. 97-106.

\section{Table 1 Summary of localization studies}

\begin{tabular}{llcl} 
GeneID/name & $\begin{array}{l}\text { TrypTag } \\
\text { PFa, endogenous }\end{array}$ & other studies & $\begin{array}{c}\text { This study } \\
\text { BF, ectopic, } \\
\text { C-terminal tag }\end{array}$ \\
\hline $\begin{array}{ll}\text { Tb927.2.2720 } \\
\text { MEKK1 }\end{array}$ & $\begin{array}{l}\text { N -faint }{ }^{\mathrm{b}} \text {, cytoplasm and flagellum; } \\
\text { C - faint, cytoplasmic, reticular }\end{array}$ & $\begin{array}{c}\text { Flagellum, flagellar pocket; } \\
\text { similar in PF }\end{array}$ \\
Tb927.3.5650 & nd & ER \\
Tb927.4.2500 & N - very faint, cytoplasmic puncta; & Flagellar pocket and \\
eIF2K2 & C - nd & nd \\
Tb927.5.3150 & N- nd; C - reticulated with & & ER \\
occasional bright puncta & & \\
Tb927.7.5220 & nd & At or near flagellar pocket, \\
FHK & similar in PF \\
Tb927.9.3120 & nd & ER \\
Tb927.9.12400 & nd & ER \\
Tb927.10.1910 & N - faint, endocytic and cytoplasm; & nd
\end{tabular}


nd

Tb92
LDK

Tb927.11.14070

RDK1
Lipid droplets [10] nd

Periphery [9]

ER

${ }^{a}$ TrypTag: in situ tags the test protein with neon-green at the N- or C-terminus of the protein for expression in PF [26].

${ }^{b}$ Faint, $10-20^{\text {th }}$ percentile of all lines expressing tagged proteins; very faint, $<10^{\text {th }}$ percentile. Note that these are all fainter than the parental line, but in cases where proteins are localized to specific organelles, localization can often be visualized.

\section{Figure Legends}

Figure 1. Schematics of $\boldsymbol{T}$. brucei protein kinases predicted to bear transmembrane domains. TMDs predicted by both TMHMM and CCTOP are marked with red bars (one predicted by TMHMM only is marked with a red X); additional TMDs predicted by CCTOP are marked by red arrowheads. Predicted signal sequences are indicated by a red asterisk. Light blue regions are ePK Pfam domains. Yellow bars depict other domains: PAS motif (P), forkhead associated domain (F), nucleotide cyclase-like domain (NTC), Kelch propeller blade (K). Light green bars demarcate regions with predicted structural similarity to known structures revealed by HHpred. Bacterial DHp, catalytic, and receiver domains characteristic of histidine kinases (D-C-R) in Tb927.9.3120, as well as bacterial sensor/receptor domains (S/R) and adenylyl/guanylyl cyclases (AGC) in RDK1, exhibited probabilities $>95 \%$ (see Figs. 2 and S2 for details). The PAS-like domain in FHK was detected with $>90 \%$ probability. Length of the predicted protein in amino acids is indicated.

Fig. 2. HHpred analysis of Tb927.9.3120 shows evidence of ancient domains. The PK domain (aa 737-980) is not shown. Red ovals - transmembrane domains predicted by TMHMM and CCTOP. Each bar below the gene model represents the top hits by HHpred. The color of the arrows mark descriptions matching the hits. The description includes the PDB number and chain designation (Hit), a description of the PDB entry including related PDB entries (Name), the probability of the hit based on the Hidden Markov Model (Probability), the probability of the match in an unrelated database (E-value), score for the secondary structure prediction (SS), number of amino acids aligned $(\mathrm{Col})$, and the total length of the target in PDB (Target Length). The documentation for HHpred considers "Probability" the most important criterion with our hits meeting at least one of the twomost stringent criteria: having a score $>95 \%$ or having a score $>50 \%$ and making reasonable biological sense [19]. 
Fig. 3. Protein production and mRNA levels of TMD PKs. Protein production was measured by ribosome profiling and mRNA levels were measured in the same biological samples (three replicates each). Data is shown as reads per $\mathrm{kb}$ and is derived from [25].

Figure 4. Localization of V5-tagged predicted membrane kinases to the ER in bloodstream form $T$. brucei. BF were fixed and stained with primary antibodies. Bound antibodies were revealed with secondary fluorochrome-coupled antibodies. Green, V5-tagged proteins, Red, anti-BiP, Blue, DAPI; Merge, all three colors. Each series shows a single deconvolved plane, except the DIC image, which is a projection. All images are shown at the same magnification. Bar, $5 \mu \mathrm{m}$.

Figure 5. Immunofluorescence localization of MEKK1-V5 (Tb927.2.2720) in T. brucei bloodstream

forms. Top, co-staining with tomato lectin (TL, green) at $4^{\circ}$, which marks the flagellar pocket. Note that punctate staining continues to the end of the flagellum. Middle, co-staining with antibody to T. brucei PIFCT3 (red), a component of the flagellar transport system. Bottom, co-staining with antibody to a K+ channel protein of T. cruzi (left, red) and with monoclonal antibody YL2 (right, red) which detects basal bodies. The latter shows a cell with two kinetoplasts and appears to be in early mitosis. Each series shows a single deconvolved plane, except DIC images, which are projection. All images, except enlargements,

532 are shown at the same magnification (bar, $5 \mu \mathrm{m}$ ). Enlargements show detail near the kDNA. N, nucleus;

$533 \mathrm{k}, \mathrm{kDNA}$.

Figure 6. Localization of MEKK1-HA to the flagellum and flagellar pocket by immunoelectron microscopy. PF expressing MEKK1 C-terminally tagged with HA epitopes were stained with anti-HA, and staining revealed by protein-A gold $10 \mathrm{~nm}$ particles. A) The gold staining is on the flagellar pocket

537 (FP) membrane, flagellum (F), but absent from the mitochondrion (m). B) staining is observed at the base of the flagellar pocket at the basal bodies (BB). C) no staining of the kinetoplast (k) is seen. D) in a dividing cell, both flagellar pockets are stained. E) the external portion of the flagellum is also stained. Bars, $100 \mathrm{~nm}$.

541 Figure 7. MEKK1 is an integral membrane protein which has protein kinase activity. A) Cell

542 fractionation. PF cells expressing MEKK1-HA were fractionated by digitonin treatment followed by

543 carbonate extraction. After separation by SDS-PAGE and blotting, the blots were probed with mouse anti-

544 HA (green) and rabbit anti-phosphoglycerate kinase (red) which detects the $56 \mathrm{kDa}$ glycosomal form

545 (PGKA) and the $47 \mathrm{kDa}$ cytosolic isoform (PGKB). A parallel blot was also incubated with antibody to

546 the acidocalcisome membrane protein vacuolar phosphatase (VP). SDS, SDS lysate; T, total after

547 incubation with digitonin; S1, digitonin supernatant; P1, digitonin pellet; S2, carbonate supernatant; P2, 548 carbonate pellet. Lanes contain fractions from $3 \times 10^{6}$ cells. B) Kinase activity. Immunoprecipitated 
MEKK1 from PF was incubated with $\gamma^{32} \mathrm{P}-\mathrm{ATP}$ and the exogenous substrate myelin basic protein (MBP), followed by SDS-PAGE and transfer to nitrocellulose. A control immunoprecipitation used the untransfected parental line (29.13). Top: phosphorimaging demonstrates phosphorylation of MBP. Bottom: the blot was probed with anti-V5 mAb demonstrating the pulldown of the tagged protein.

Figure 8. Predominant localization of epitope-tagged FHK near the kinetoplast. A, B) BF expressing FHK-V5 and co-stained with DAPI, anti-V5 antibodies, and either anti-PIFTC3 (A) or anti-BiP (B). Enlargement shows region around kDNA. The cell in (A) has duplicated both its nucleus (N) and kDNA (k) and is undergoing cytokinesis as evidenced by the presence of two flagella (F). The cell in panel B has an elongated, slightly $\mathrm{V}$-shaped kinetoplast, indicating it is undergoing kinetoplast division and is in nuclear S phase. Note that a point of FHK-staining occurs at either pole of the elongated kDNA. C) PF expressing FHK-HA were incubated with biotinylated-tomato lectin (TL) at $4^{\circ}$ and fixed prior to staining with DAPI, avidin and anti-HA. Enlargement shows region around kDNA. D) Whole cell and enlarged serial planes (numbered) of a DAPI-stained PF cell expressing FHK-HA. All images (except enlargements) are at the same magnification. Bar $=5 \mu \mathrm{m}$.

\section{Fig. 9. Localization of FHK-HA to the flagellar pocket by immunoelectron microscopy}

Procyclic form parasites expressing FHK C-terminally tagged with HA epitopes were stained with antiHA revealed by protein-A gold $10 \mathrm{~nm}$ particles. A) The gold staining is on the flagellar pocket (FP) membrane but absent from the flagellum (F) and mitochondrion (m). Enlargement of the marked region is shown in panel a. B) Staining is predominantly at the flagellar pocket, and often one side shows more abundant FHK. Enlargement of the marked region is shown in panel b. C) The staining is sometimes seen on vesicles close to the FP. D) Occasional parasites showed staining outside the flagellar pocket. Bars, $100 \mathrm{~nm}$.

\section{Figure 10. FHK is an integral membrane protein with protein kinase activity. A) Cell fractionation} and immunoblotting were as described in Fig. 7. B) Kinase activity as described in Fig. 9. FHK-HA was immunoprecipitated from PF and incubated with $\gamma^{32} \mathrm{P}$-ATP and MBP. A negative control immunoprecipitation was performed using the untransfected parental line 29.13. Top: phosphorimaging demonstrates phosphorylation of MBP. Bottom: the blot was probed with anti-HA mAb demonstrating the pulldown of the tagged protein.

\section{List of supplementary information}

\section{Supplementary tables}


580 Table S1. TriTryp orthologues - transmembrane domains, signal sequences, and additional

581 domains

582 Table S2. Primers

\section{Supplementary figures}

584 Fig. S1. RDK1 structural similarities identified by HHpred

585 Figure S2. Western blot showing expression of epitope-tagged PKs in T. brucei bloodstream 586 forms.

587

588

589 


\section{Figure 1}

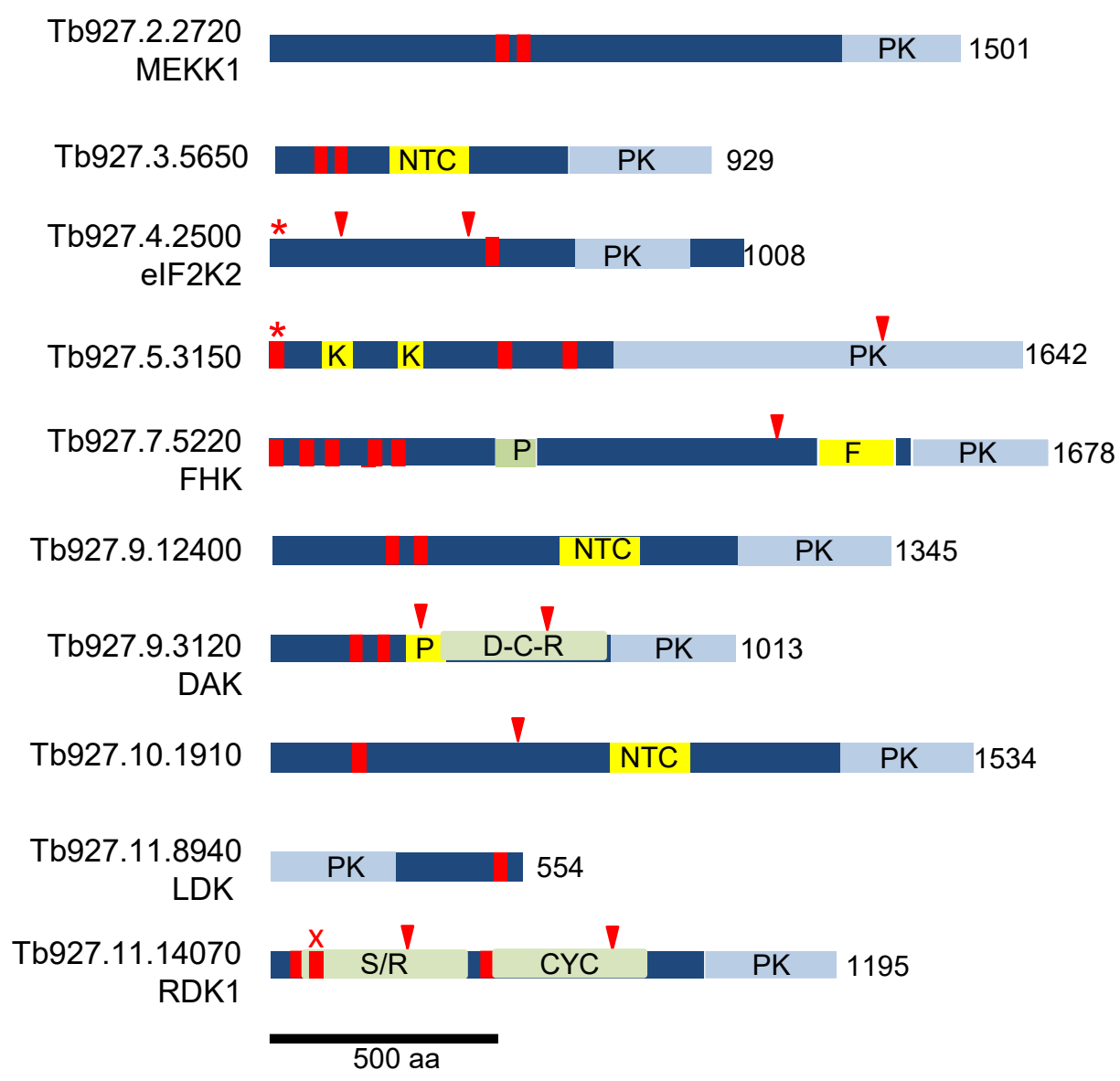

Figure 1. Schematics of $T$. brucei protein kinases predicted to bear transmembrane domains. TMDs predicted by both TMHMM and CCTOP are marked with red bars (one predicted by TMHMM only is marked with a red X); additional TMDs predicted by CCTOP are marked by red arrowheads. Predicted signal sequences are indicated by a red asterisk. Light blue regions are ePK Pfam domains. Yellow bars depict other domains: PAS motif $(P)$, forkhead associated domain (F), nucleotide cyclase-like domain (NTC), Kelch propeller blade (K). Light green bars demarcate regions with predicted structural similarity to known structures revealed by HHpred. Bacterial DHp, catalytic, and receiver domains characteristic of histidine kinases (D-C-R) in Tb927.9.3120, as well as bacterial sensor/receptor domains $(\mathrm{S} / \mathrm{R}$ ) and adenylyl/guanylyl cyclases (AGC) in RDK1, exhibited probabilities $>95 \%$ (see Figs. 2 and S2 for details). The PAS-like domain in FHK was detected with $>90 \%$ probability. Length of the predicted protein in amino acids is indicated. 


\section{Figure 2}

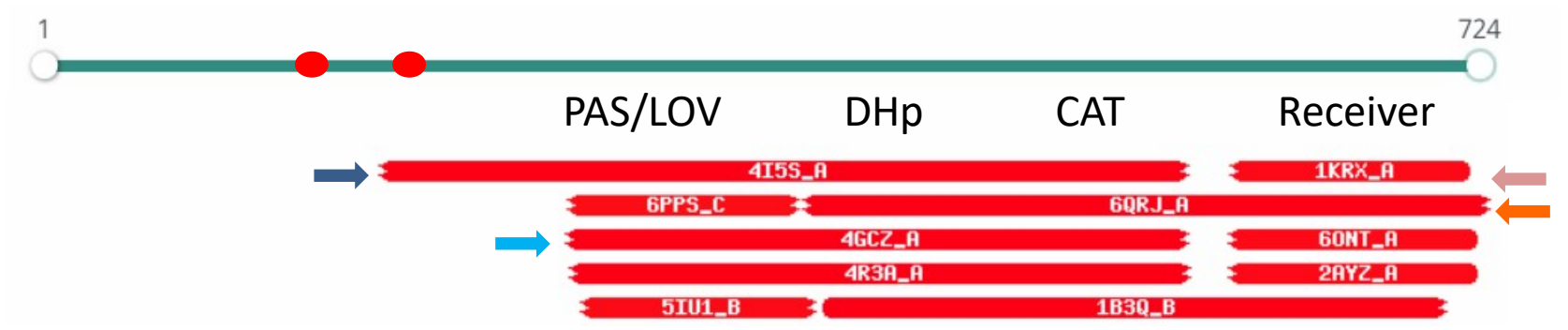

\begin{tabular}{|c|c|c|c|c|c|c|c|c|}
\hline & $\mathrm{Nr}$ & Hit & Name & Probability ${ }^{\overrightarrow{ }}$ & E-value $\hat{}$ & ss $\stackrel{\Delta}{*}$ & Cols $^{\hat{v}}$ & $\begin{array}{l}\text { Target } \\
\text { Length }\end{array}$ \\
\hline$\longrightarrow$ & 1 & 4I5S_A & $\begin{array}{l}\text { Putative histidine kinase CovS ; HISTIDINE KINASE, VICK, } \\
\text { HAMP, PAS; } 3.3 \mathrm{~A} \text { \{Streptococcus mutans serotype c\}; Related } \\
\text { PDB entries: } 4 \text { I5S_B }\end{array}$ & 99.95 & $2.2 \mathrm{e}-26$ & 25.8 & 372 & 450 \\
\hline$\Rightarrow$ & 02 & 6QRJ_A & $\begin{array}{l}\text { Hybrid kinase; Cyclic di-GMP, second messenger, hybride; } \\
\text { HET: ANP; } 2.65 \text { ACaulobacter vibrioides CB15\} }\end{array}$ & 99.94 & $1.5 e-24$ & 29.6 & 301 & 484 \\
\hline$\Rightarrow$ & 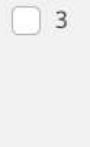 & 4GCZ_A & $\begin{array}{l}\text { Blue-light photoreceptor, Sensor protein fixL; photoreceptor, } \\
\text { signal transduction, two-component system; HET: MSE, SO4, } \\
\text { ADP, FMN; } 2.3 \mathrm{~A}\{\text { Bacillus subtilis\}; Related PDB entries: } \\
\text { 4GCZ_B }\end{array}$ & 99.89 & $8.6 \mathrm{e}-21$ & 31.8 & 294 & 385 \\
\hline & 104 & 4R3A_A & $\begin{array}{l}\text { Blue-light-activated histidine kinase } 2 \text { (E.C.2.7.13.3); light- } \\
\text { activated, LOV domain, histidine kinase; HET: RBF, ANP; } 2.92 \text { A } \\
\text { \{Erythrobacter litoralis HTCC2594\}; Related PDB entries: } \\
\text { 4R3A_B }\end{array}$ & 99.88 & $2.8 \mathrm{e}-20$ & 31.5 & 296 & 352 \\
\hline & 05 & 1B3Q_B & $\begin{array}{l}\text { CHEMOTAXIS PROTEIN CHEA; HISTINE KINASE, SIGNAL } \\
\text { TRANSDUCTION, CHEMOTAXIS; 2.6A \{Thermotoga maritima\} } \\
\text { SCOP: d.122.1.3, a.30.2.1, b.40.7.1; Related PDB entries: } \\
\text { 1B3Q_A 3JA6_C 3JA6_E }\end{array}$ & 99.88 & $9.6 \mathrm{e}-22$ & 19 & 281 & 379 \\
\hline$\Rightarrow$ & 42 & $1 \mathrm{KRXA}$ & $\begin{array}{l}\text { NITROGEN REGULATION PROTEIN NR(I); two component } \\
\text { signal transduction, receiver; HET: BEF; NMR \{Salmonella } \\
\text { typhimurium\} SCOP: c.23.1.1; Related PDB entries: 1DC7_A } \\
\text { 1DC8_A 1556_A 1KRW_A 2MSK_A 2MSL_A 1NTR_A }\end{array}$ & 98.73 & $2.3 e-7$ & 13.2 & 114 & 124 \\
\hline
\end{tabular}

Fig. 2. HHpred analysis of Tb927.9.3120 shows evidence of ancient domains. The PK domain (aa 737-980) is not shown. Red ovals - transmembrane domains predicted by TMHMM and CCTOP. Each bar below the gene model represents the top hits by HHpred. The color of the arrows mark descriptions matching the hits. The description includes the PDB number and chain designation (Hit), a description of the PDB entry including related PDB entries (Name), the probability of the hit based on the Hidden Markov Model (Probability), the probability of the match in an unrelated database (E-value), score for the secondary structure prediction (SS), number of amino acids aligned (Col), and the total length of the target in PDB (Target Length). The documentation for HHpred considers "Probability" the most important criterion with our hits meeting at least one of the twomost stringent criteria: having a score $>95 \%$ or having a score $>50 \%$ and making reasonable biological sense [19]. 


\section{Figure 3}

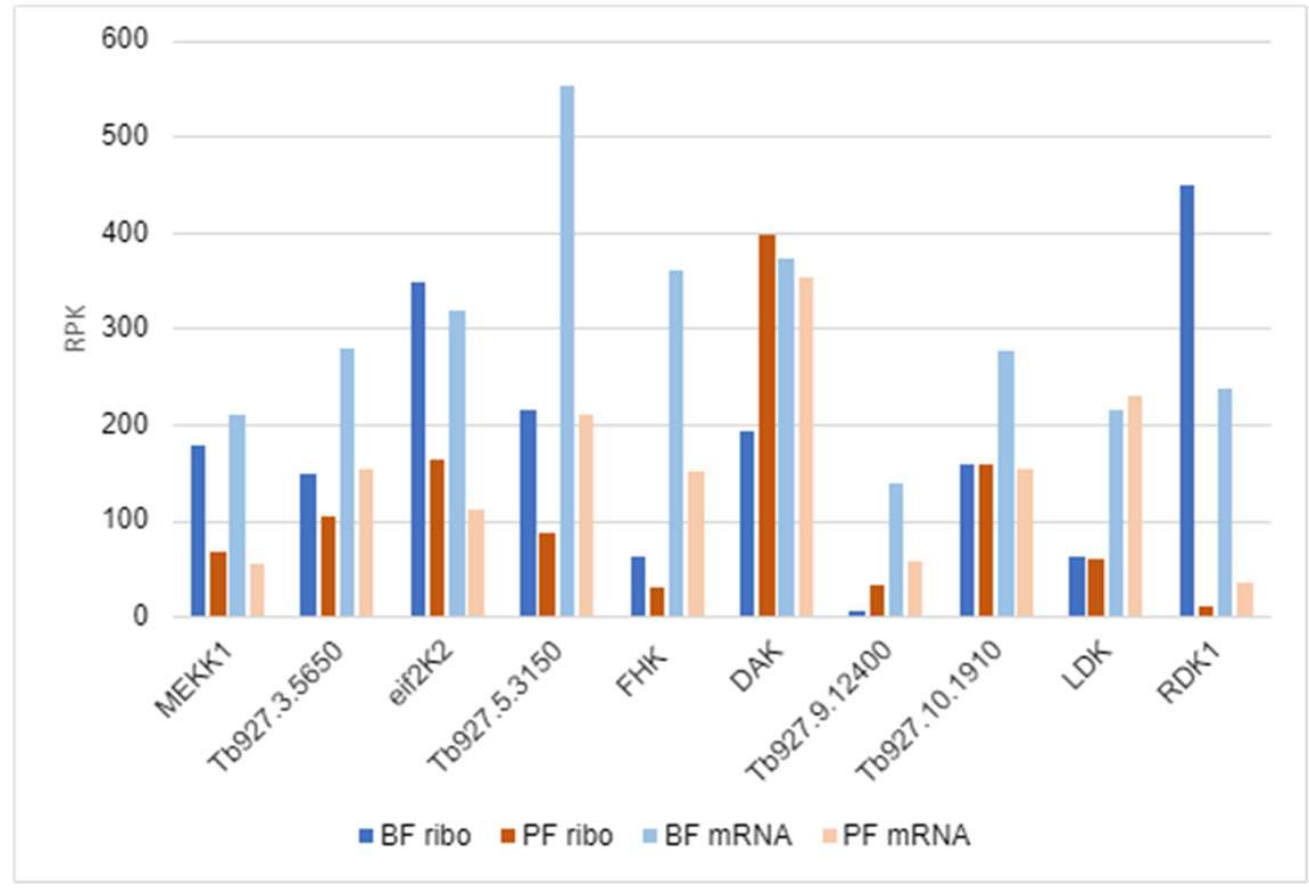

Fig. 3. Protein production and mRNA levels of TMD PKs. Protein production was measured by ribosome profiling and mRNA levels were measured in the same biological samples (three replicates each). Data is shown as reads per kb and is derived from [25]. 


\section{Figure 4}

DIC

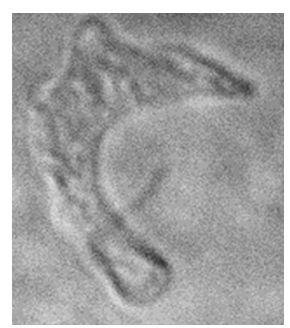

Tb927.3.5650

Tb927.5.3150

Tb927.9.3120

DAK

Tb927.9.12400
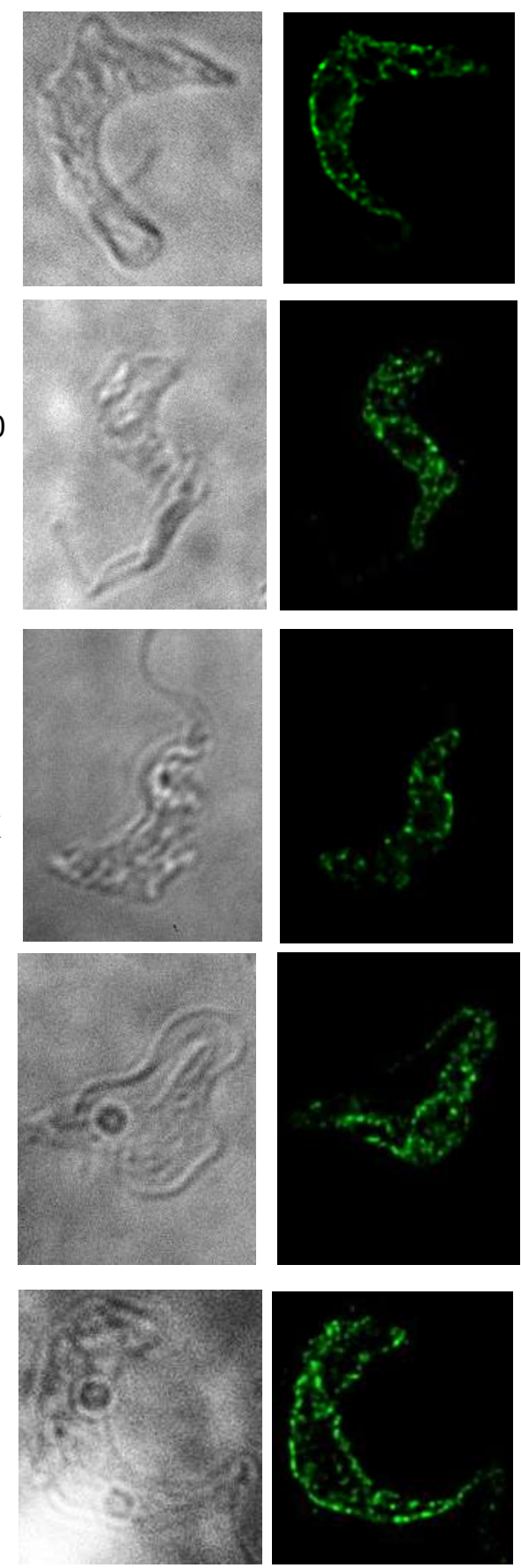
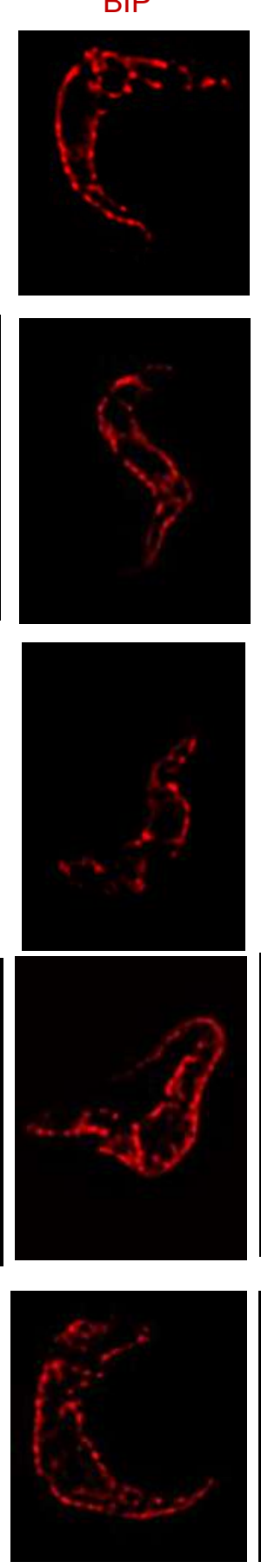

DAPI
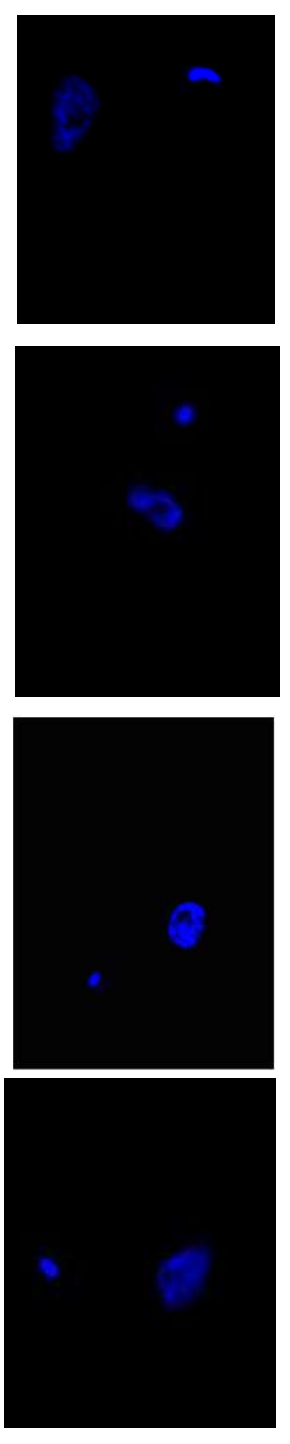

Merge
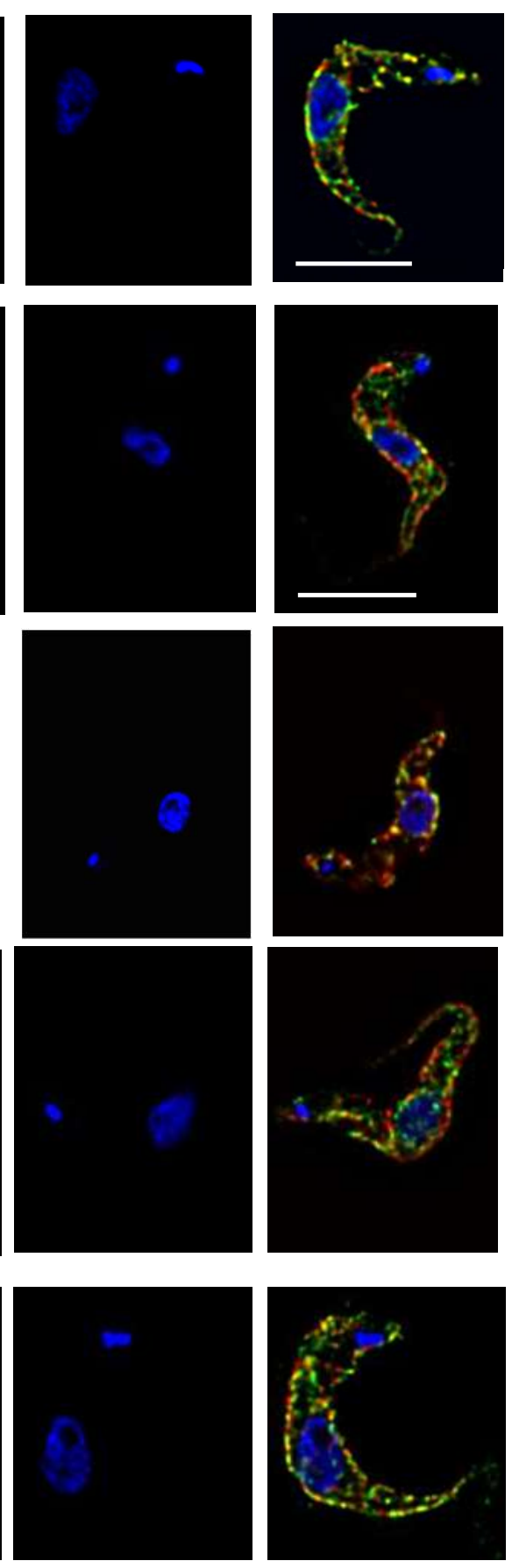

Figure 4. Localization of V5-tagged predicted membrane kinases to the ER in bloodstream form $T$. brucei. BF were fixed and stained with primary antibodies. Bound antibodies were revealed with secondary fluorochrome-coupled antibodies. Green, V5tagged proteins, Red, anti-BiP, Blue, DAPI; Merge, all three colors. Each series shows a single deconvolved plane, except the DIC image, which is a projection. All images are shown at the same magnification. Bar, $5 \mu \mathrm{m}$. 


\section{Figure 5}
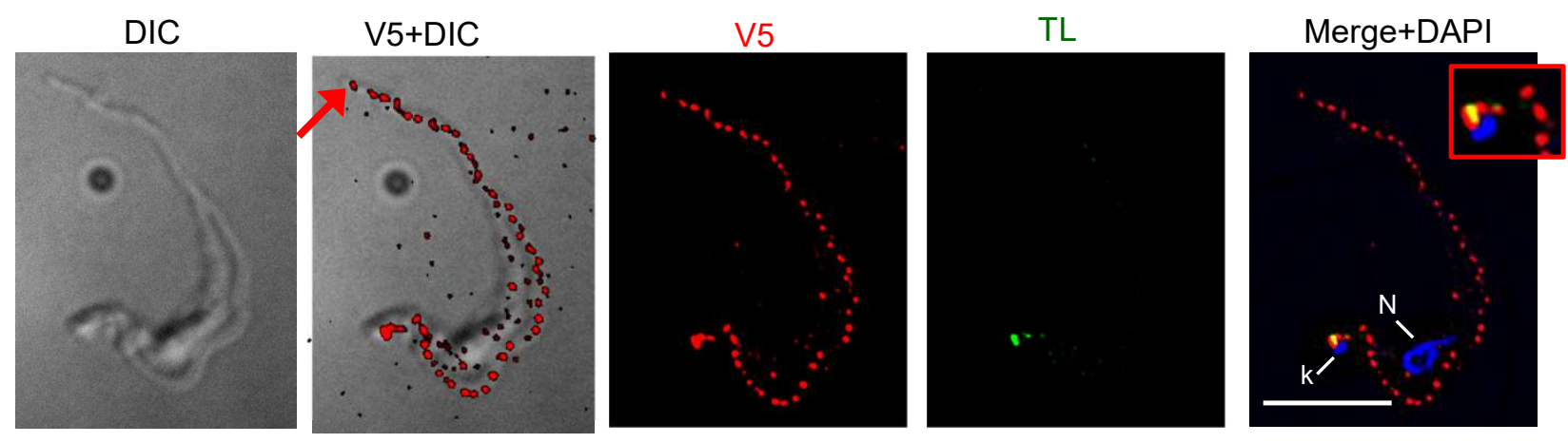

DIC

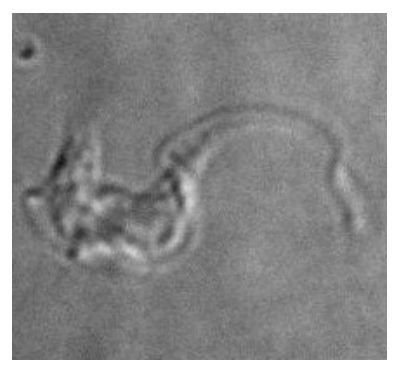

V5
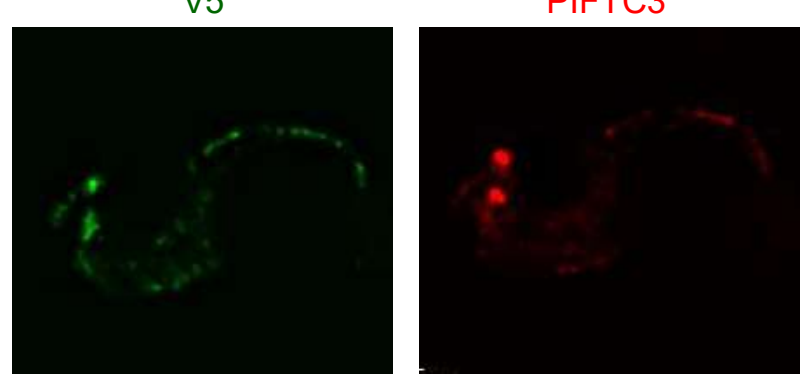

DIC
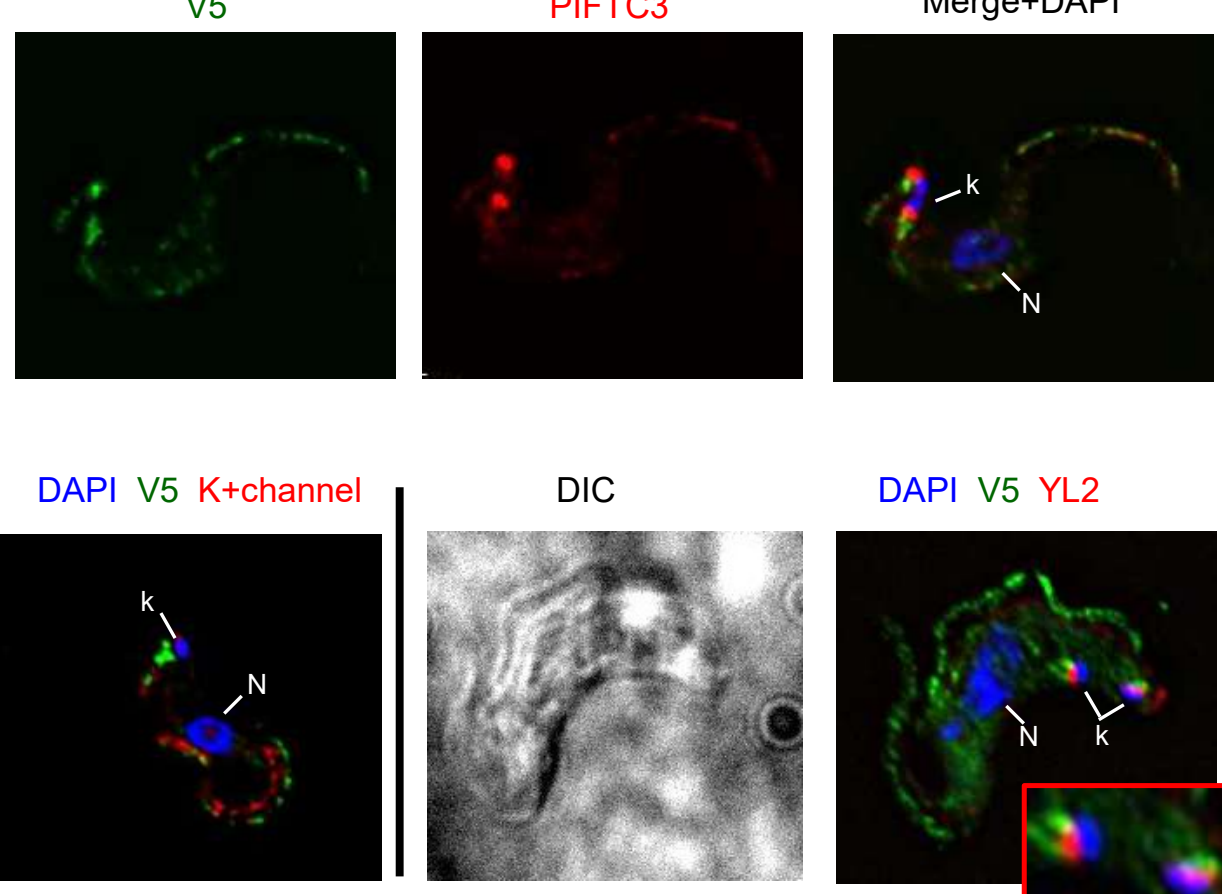

DIC

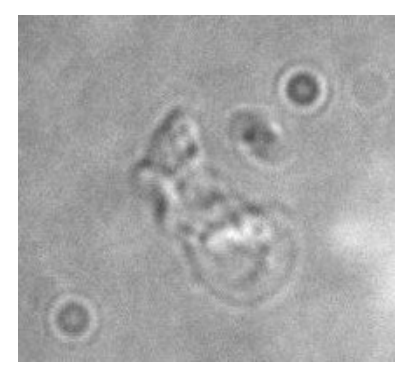

DAPI V5 YL2

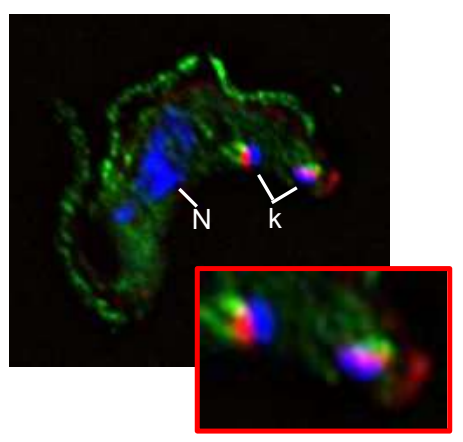

Figure 5. Immunofluorescence localization of MEKK1-V5 (Tb927.2.2720) in $T$. brucei bloodstream forms. Top, co-staining with tomato lectin (TL, green) at $4^{\circ}$, which marks the flagellar pocket. Note that punctate staining continues to the end of the flagellum. Middle, co-staining with antibody to T. brucei PIFCT3 (red), a component of the flagellar transport system. Bottom, co-staining with antibody to a $\mathrm{K}+$ channel protein of T. cruzi (left, red) and with monoclonal antibody YL2 (right, red) which detects basal bodies. The latter shows a cell with two kinetoplasts and appears to be in early mitosis. Each series shows a single deconvolved plane, except DIC images, which are projection. All images, except enlargements, are shown at the same magnification (bar, $5 \mu \mathrm{m}$ ). Enlargements show detail near the kDNA. N, nucleus; $\mathrm{k}$, kDNA. 


\section{Figure 6}

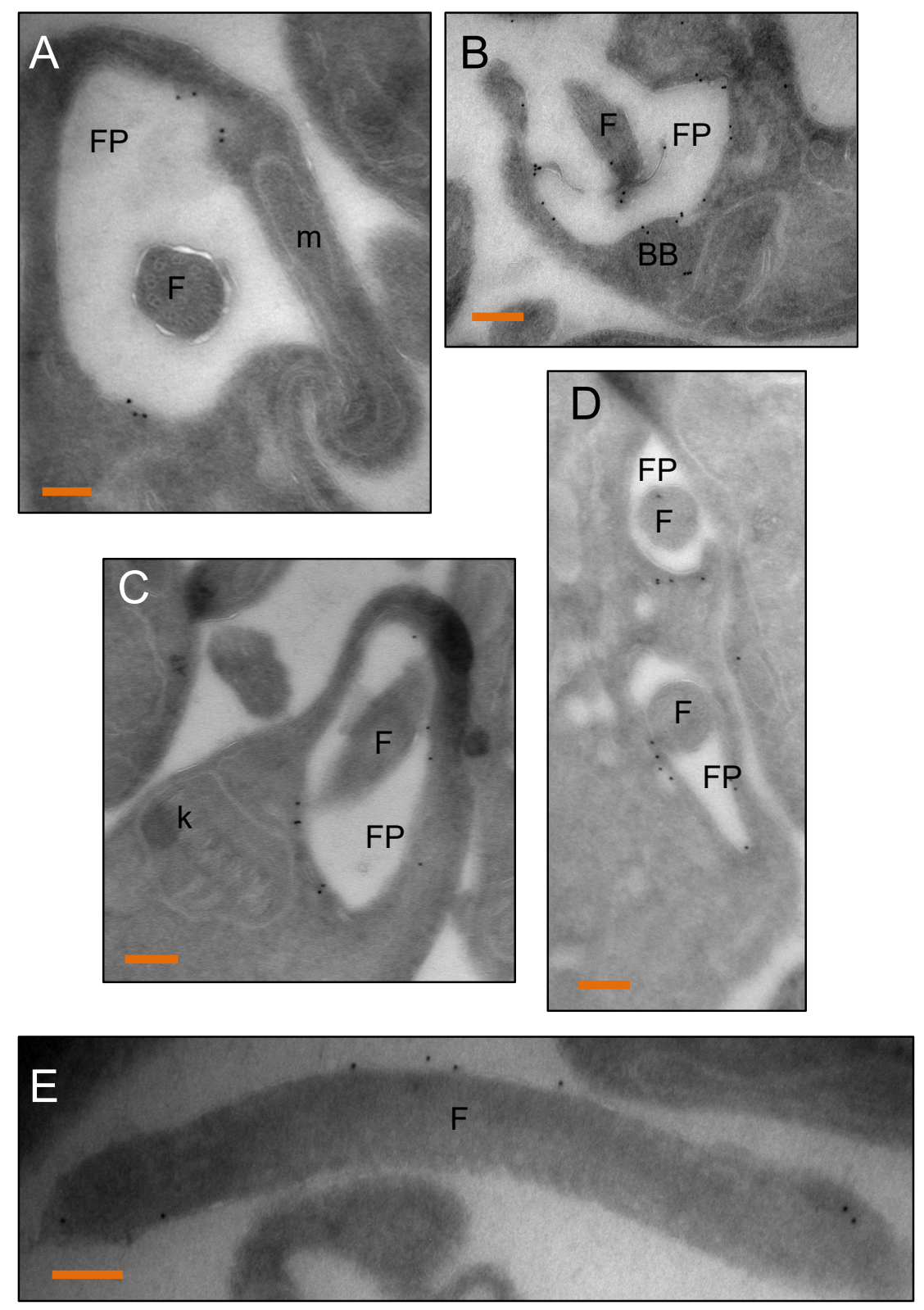

Figure 6. Localization of MEKK1-HA to the flagellum and flagellar pocket by immunoelectron microscopy. PF expressing MEKK1 C-terminally tagged with HA epitopes were stained with anti-HA, and staining revealed by protein-A gold $10 \mathrm{~nm}$ particles. A) The gold staining is on the flagellar pocket (FP) membrane, flagellum (F), but absent from the mitochondrion $(m)$. B) staining is observed at the base of the flagellar pocket at the basal bodies (BB). C) no staining of the kinetoplast (k) is seen. D) in a dividing cell, both flagellar pockets are stained. E) the external portion of the flagellum is also stained. Bars, $100 \mathrm{~nm}$. 


\section{Figure 7}

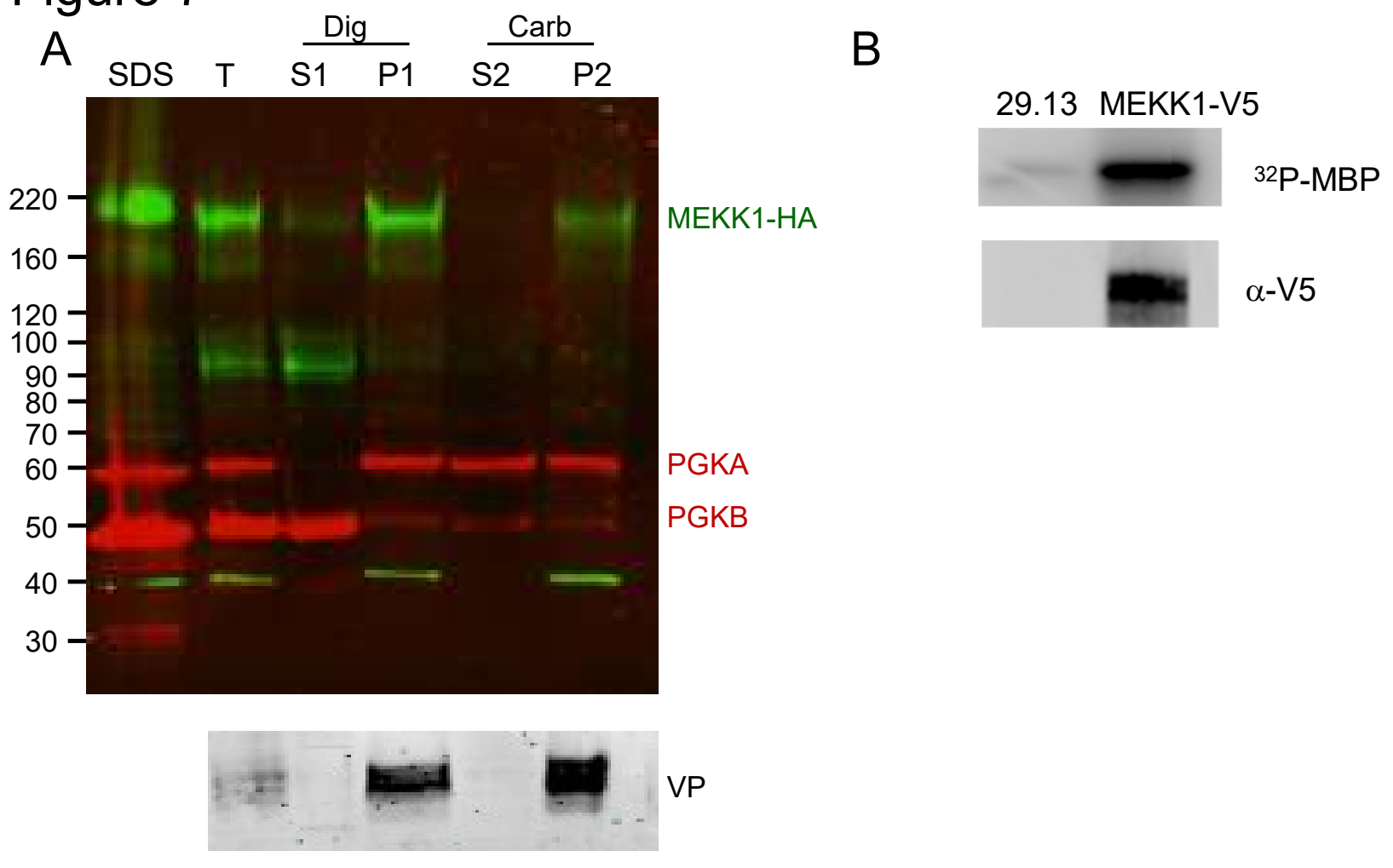

Figure 7. MEKK1 is an integral membrane protein which has protein kinase activity.

A) Cell fractionation. PF cells expressing MEKK1-HA were fractionated by digitonin treatment followed by carbonate extraction. After separation by SDS-PAGE and blotting, the blots were probed with mouse anti-HA (green) and rabbit anti-phosphoglycerate kinase (red) which detects the $56 \mathrm{kDa}$ glycosomal form (PGKA) and the $47 \mathrm{kDa}$ cytosolic isoform (PGKB). A parallel blot was also incubated with antibody to the acidocalcisome membrane protein vacuolar phosphatase (VP). SDS, SDS lysate; T, total after incubation with digitonin; S1, digitonin supernatant; P1, digitonin pellet; S2, carbonate supernatant; P2, carbonate pellet. Lanes contain fractions from $3 \times 106$ cells. B) Kinase activity. Immunoprecipitated MEKK1 from PF was incubated with y 32P-ATP and the exogenous substrate myelin basic protein (MBP), followed by SDS-PAGE and transfer to nitrocellulose. A control immunoprecipitation used the untransfected parental line (29.13). Top: phosphorimaging demonstrates phosphorylation of MBP. Bottom: the blot was probed with anti-V5 $\mathrm{mAb}$ demonstrating the pulldown of the tagged protein. 


\section{Figure 8}

A DIC

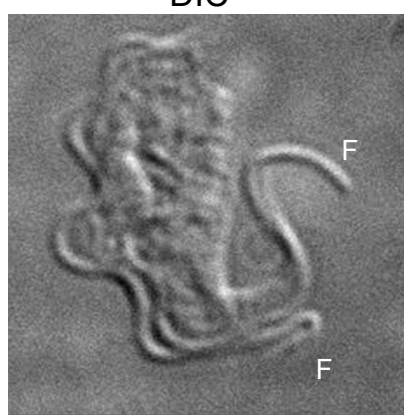

B

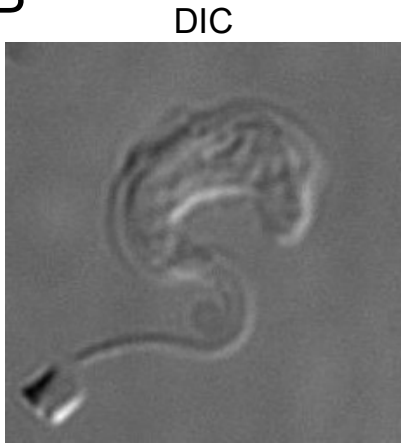

C

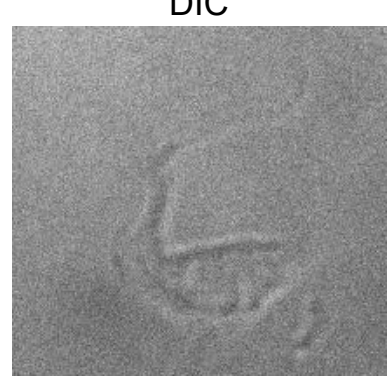

V5

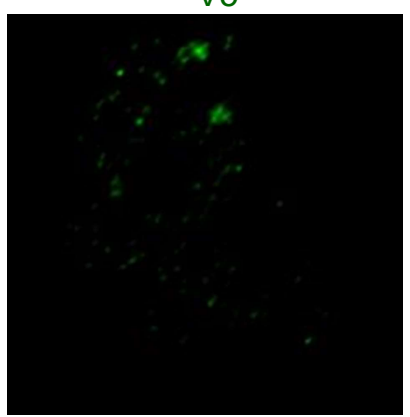

V5

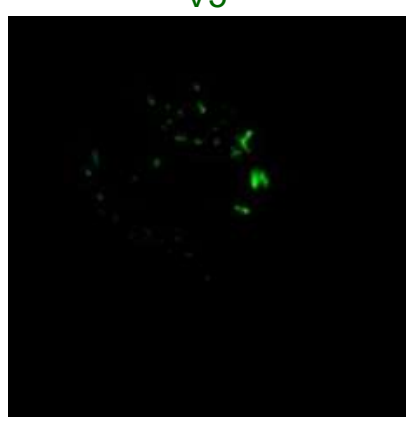

$\mathrm{HA}+\mathrm{DAPI}$
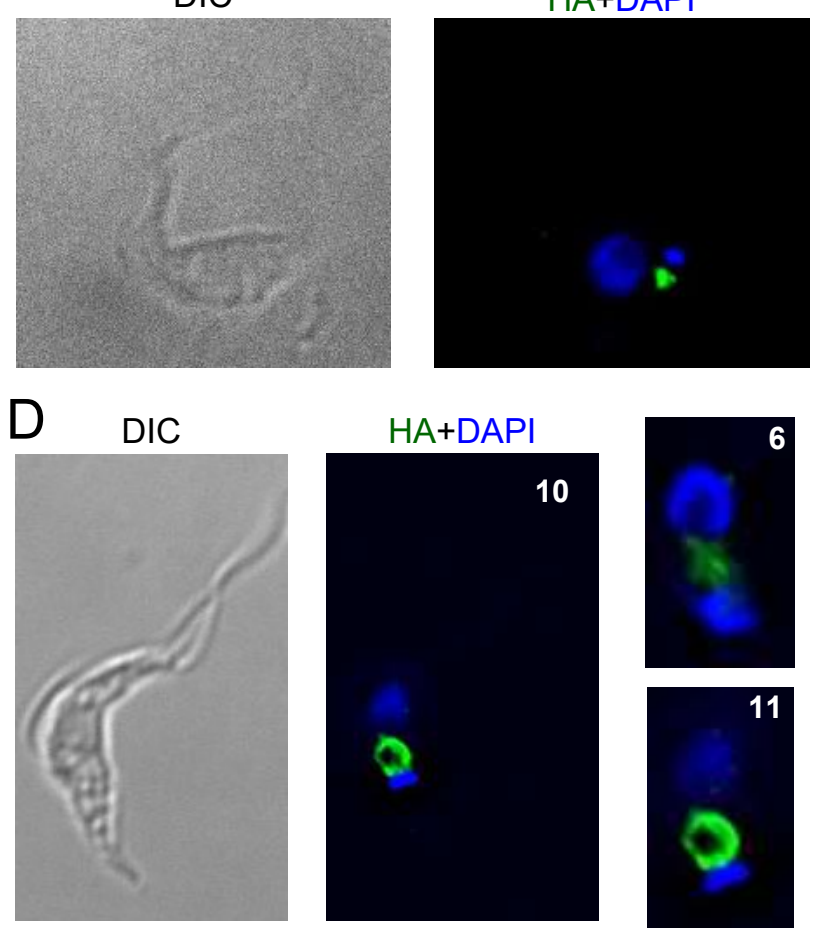

$H A+D A P I$

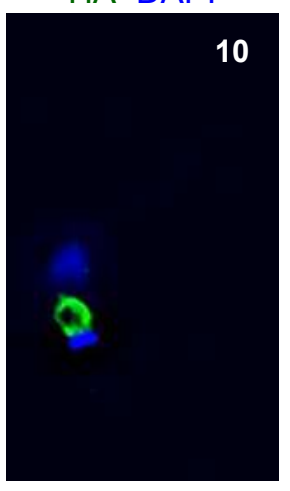

PIFTC3

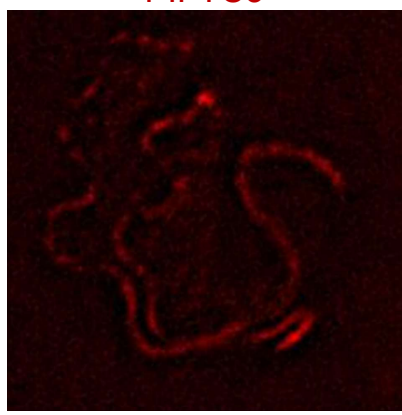

$\mathrm{BiP}$

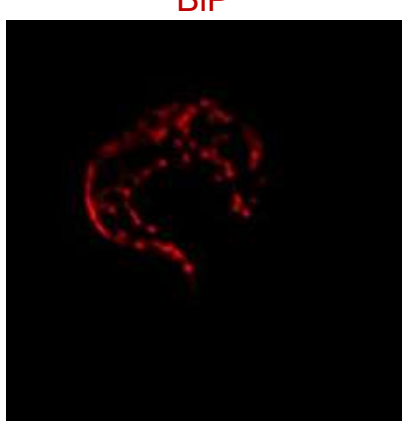

$T L+\mathrm{DAPI}$

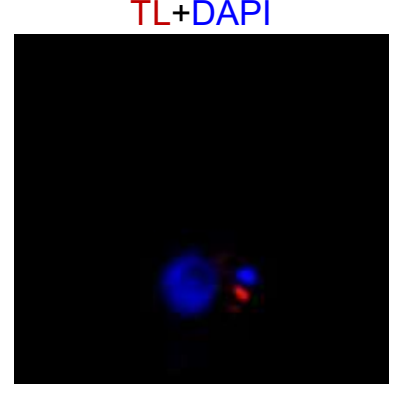

7
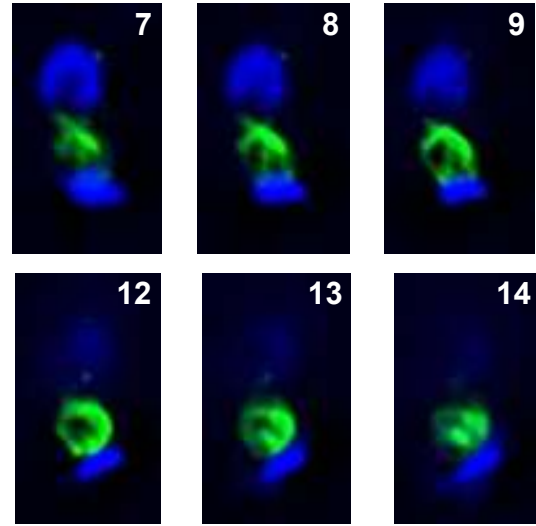

9
Merge+DAPI

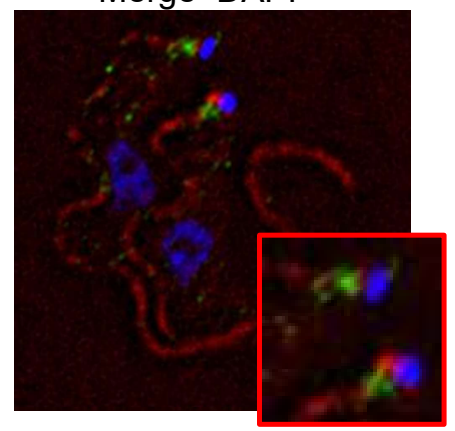

Merge+DAPI
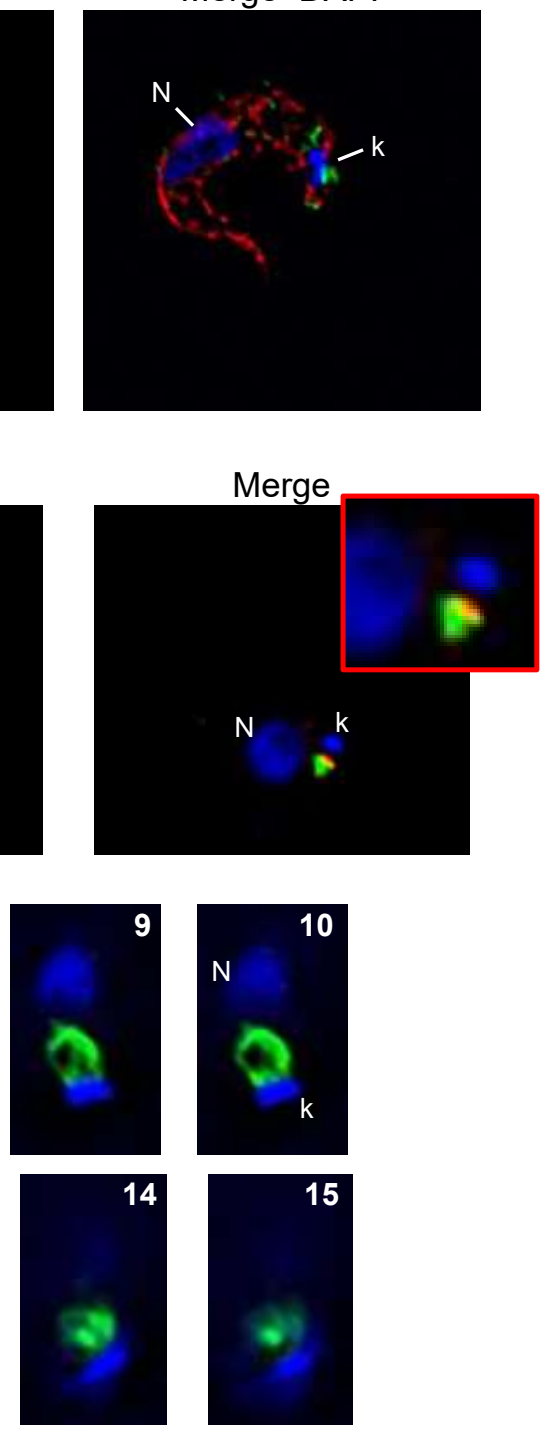

Figure 8. Predominant localization of epitope- tagged FHK near the kinetoplast. A, B) BF expressing FHKV5 and co-stained with DAPI, anti-V5 antibodies, and either anti-PIFTC3 (A) or anti-BiP (B). Enlargement shows region around $k D N A$. The cell in $(A)$ has duplicated both its nucleus $(N)$ and $k D N A(k)$ and is undergoing cytokinesis as evidenced by the presence of two flagella $(F)$. The cell in panel $B$ has an elongated, slightly $V$ shaped kinetoplast, indicating it is undergoing kinetoplast division and is in nuclear $S$ phase. Note that a point of FHK-staining occurs at either pole of the elongated kDNA. C) PF expressing FHK-HA were incubated with biotinylated-tomato lectin (TL) at 40 and fixed prior to staining with DAPI, avidin and anti-HA. Enlargement shows region around kDNA. D) Whole cell and enlarged serial planes (numbered) of a DAPI-stained PF cell expressing FHK-HA. All images (except enlargements) are at the same magnification. Bar $=5 \mu \mathrm{m}$. 
bioRxiv preprint doi: https://doi.org/10.1101/2020.12.08.416529; this version posted December 22, 2020. The copyright holder for this preprint

\section{Figure 9}
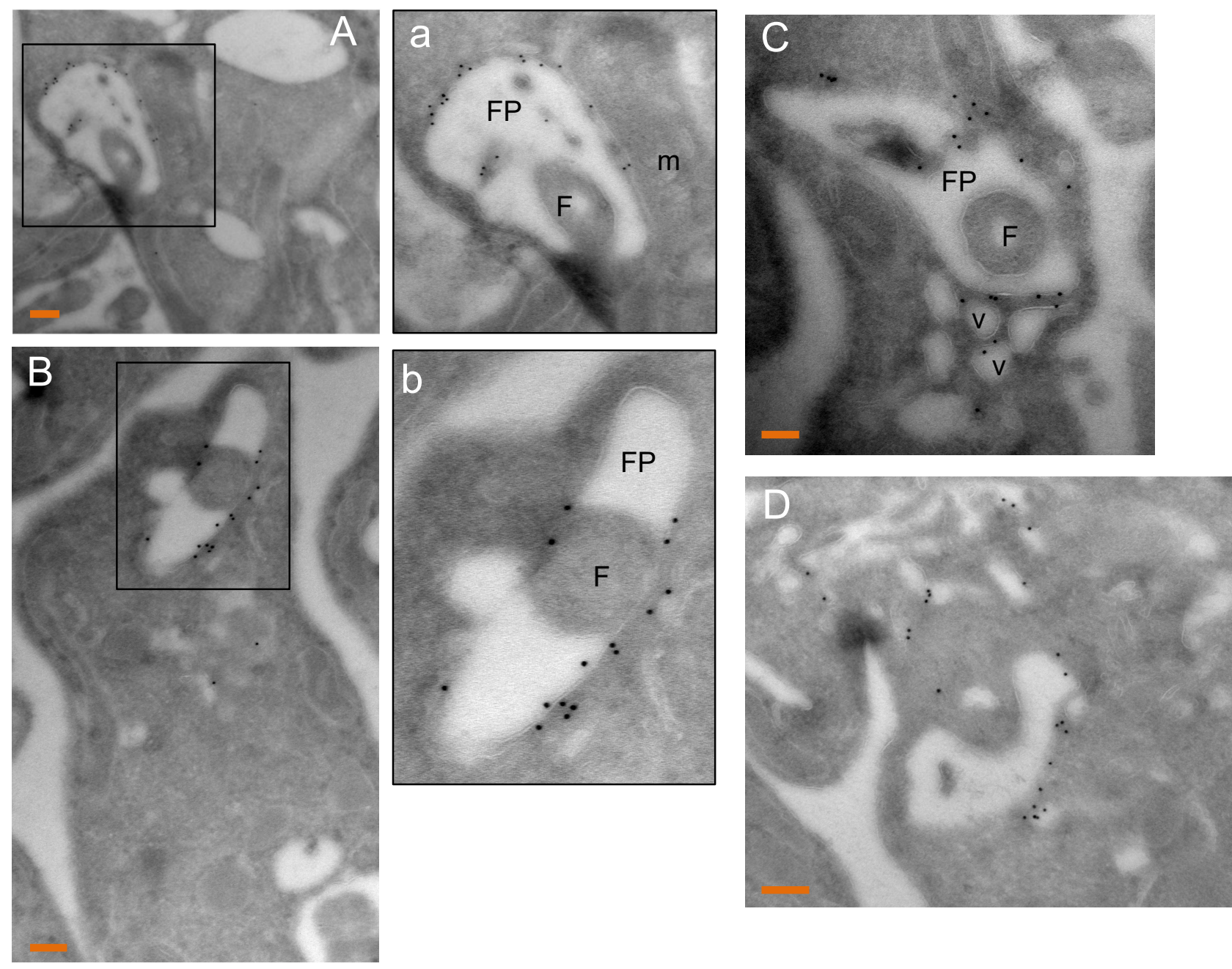

Fig. 9. Localization of FHK-HA to the flagellar pocket by immunoelectron microscopy

Procyclic form parasites expressing FHK C-terminally tagged with HA epitopes were stained with anti-HA revealed by protein-A gold $10 \mathrm{~nm}$ particles. A) The gold staining is on the flagellar pocket (FP) membrane but absent from the flagellum $(F)$ and mitochondrion $(m)$. Enlargement of the marked region is shown in panel a. B) Staining is predominantly at the flagellar pocket, and often one side shows more abundant FHK. Enlargement of the marked region is shown in panel b. C) The staining is sometimes seen on vesicles close to the FP. D) Occasional parasites showed staining outside the flagellar pocket. Bars, 100 $\mathrm{nm}$. 


\section{Figure 10}

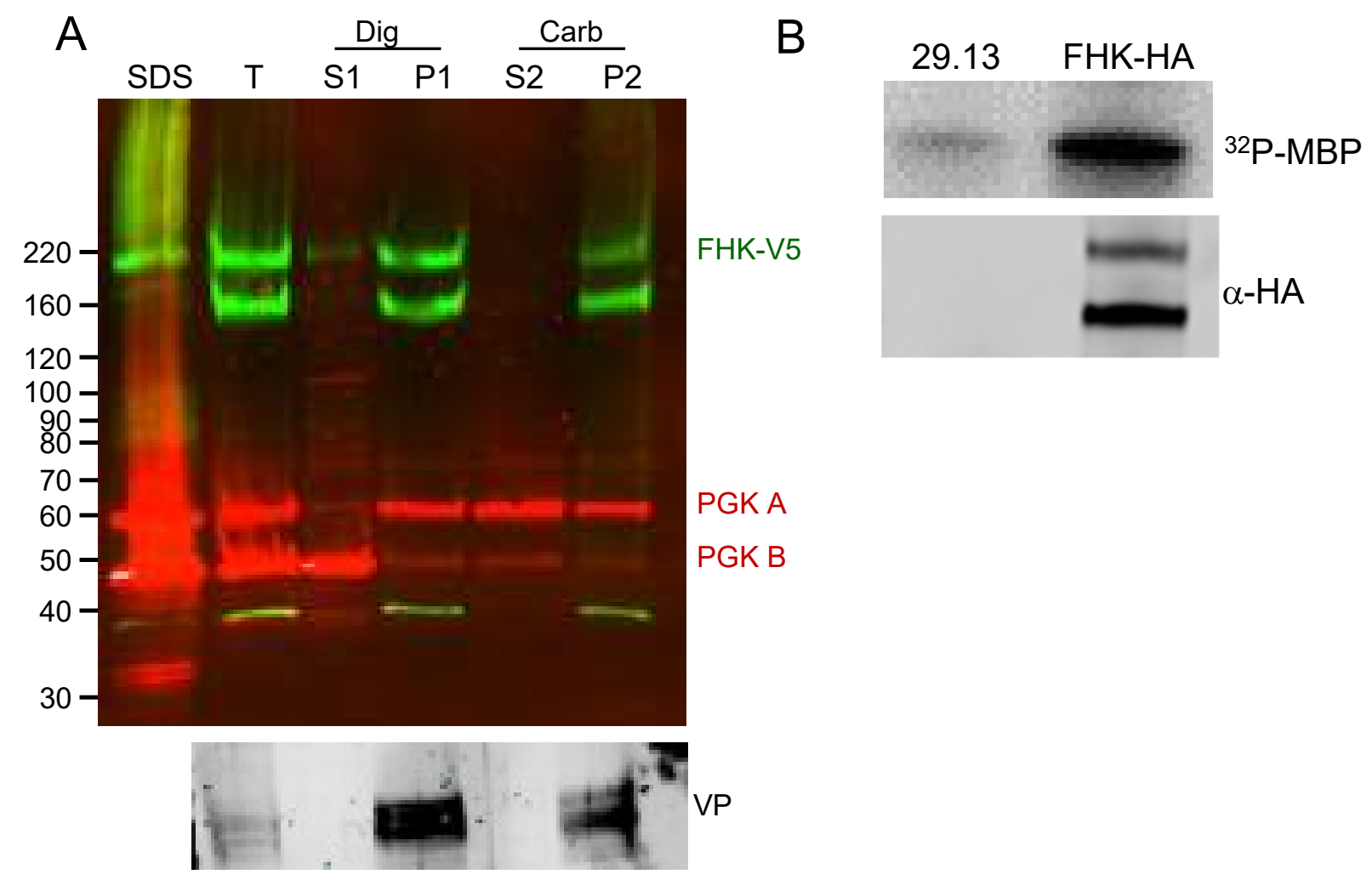

Figure 10. FHK is an integral membrane protein with protein kinase activity.

A) Cell fractionation and immunoblotting were as described in Fig. 7. B) Kinase activity as described in Fig. 9. FHK-HA was immunoprecipitated from PF and incubated with y 32PATP and MBP. A negative control immunoprecipitation was performed using the untransfected parental line 29.13. Top: phosphorimaging demonstrates phosphorylation of MBP. Bottom: the blot was probed with anti-HA mAb demonstrating the pulldown of the tagged protein. 